[Aus der Prosektur des Militärreservespitals Nr. 2 in Pardubitz (Böhmen).]

\title{
Bakteriologische Untersuchungen am pathol.-anatomischen Material von Bazillenruhr. Von
}

\author{
Dr. Hermann Šikl, \\ Assistent am böhm. pathol.-anatomischen Institut in Prag.
}

Bei Ołpduktionen von Kriegsteilnehmern begegnet man sehr oft, jedenfalles viel öfter, als es bei dem Friedensmaterial der Fall war, schweren Veränderungen der Dickdarmschleimhaut; das kann nicht wundernehmen, denn die Ruhr, auf welche solche Darmaffektionen in den meisten Fällen zurïckzuführen sind, erfuhr im Verlaufe des Krieges eine starke Verbreitung. Doch ist die Deutung dieser Befunde nicht immer einfach, hauptsächlich dann nicht, wenn man sich die Frage nach ihrer Ätiologie stellt.

Einige seltenen, mit. ähnlichem Darmbefund einhergehenden Krankheitsprozesse toxischer Natur, wie urämische, septische und Quecksilberenteritis, sind meist nicht schwer zu unterscheiden; dasselbe gilt um so mehr von tuberkulösen und luetischen Darmgeschwïren. Ziemlich scharf läßt sich weiter auch die in unserem Klima wohl selten in Betracht kommende Amöbendysentérie sowohl ätiologisch wie auch anatomisch abgrenzen (Kuenen, Löhlein).

Die übrigen Đarmprozesse fallen in den Bereich der sogenannten Bazillenruhr. Die Ätiologie dieser Krankheit durfte vor dem Kriege durch Arbeiten zahlreicher Autoren, von denen ich nur Shiga, Kruse, Flexner, Strong, Hiss und Russel sowie Lentz nennen möchte, für aufgeklärt gelten. Nun haben aber die Erfahrungen der Kriegsjahre manche Verwirrung auf diesem Felde mit sich gebracht, und die Ätiologie der Ruhr ist neuerdings zum Gegenstand vieler Diskussionen geworden 
Es hat sich nämlich gezeigt, daß die bakteriologische Stuhluntersuchung bei Ruhrkranken sehr oft versagt; das Fahnden nach den als Ruhrerreger anerkannten Bakterien brachte in vielen Fällen, ja in ganzen Epidemien negative Resultate. Welch Wunder, daß sich dadurch viele Autoren bewogen sahen, eine einheitliche Ätiologie der Ruhr überhaupt abzusprechen und wenigstens für ihre Fälle und Epidemien entweder einen unbekannten Erreger anzunehmen (Dorendorf und Kolle) oder andere in den. Stuhlprobon vorgefundene Mikroorganismen, wie Streptokokken (Menzer, Alter), Kapselbakterien (Czaplewski), Amöben (Popper) u. a. als Erreger anzuschuldigen oder schließlich toxische und physikalische Momente verschiedenster Art für die Entstehung der Ruhr verantwortlich zu machen. Andere zweifeln wieder die Spezilität der Ruhrbazillen an; unter Einwirkung von äußeren Sehädigungen, wie Strapazen, Abkühlung, Diätfehler, einseitige Kost, sogar auch psychischer Depression (Köhlisøh), sollen saprophytische Darmbakterien pathogene Eigenschaften entfalten können oder sich mehr oder weniger rasch in echte Ruhrbazillen umwandeln (Kindborg, Köhlisch, Marx, Hirseh, Falta und Kohn, Seligmann).

Prozentuelle Angaben über. positive Stuhlbefunde quoad Ruhrbazillen bewegen sich bei versehiedenen Untersuchern zwischen 0 und 100. Die Konsequenzen aber, welche daraus die-Kliniker für die Abgrenzung des Ruhrbegriffes und die Diágnose gezogen haben, sind zweierlei Art.

Dar eine Teil will nur die Fälle mit aufgeklärter Ätiologie als echte Ruhr auffassen, die uibrigen mit Durchfall und schleimig-blutigem Stuhl einhergehenden Erkrankungen bei negativem bakteriologischen Befund als "Colitis haemorrhagica" auf die oben besprochene mannigfaltige, nichtspezifische Ätiologie zurückführen (Marx, Arneth, Handmann, Grundmann u. a.).

Andere sprechen dagegen der Bakteriologie jede Bedeutung für die Diagnosestellung . $a b$ und fassen den Begriff "Ruhr" rein klinisch auf (Jürgens, Galambos, Singer).

Wenn man auch nicht von der Han'd weisen kann, dab auch andere Ursachen als Ruhrbazillen blutig-sehleimige Stühle bewirken können, so muß doch gegen die erstgenannte Ansicht eingewendet werden, daß ein negativer Stublbefund doch keineswegs das Nichtvorhandensein von Ruhrbazillen beweist; die Ursachen der Mißerfolge sind vielmehr, wenigstens zum großen Teil, in den Mängeln der bakteriologisehen Technik zu suchen. Gegen die zweitgenannte Auffassung sprechen die nicht seltenen atypischen, unter dem. Bilde einfacher Darmkatarrhe verlaufenden Fälle, die aber sowohl dureh den epidemischen Zusammenhang wie auch.auf 
Grund eines positiven Bazillenbefundes als Ruhrinfekte aufgefaßt werden müssen. Jürgens gibt selbst $z u, d a ß$ derartige Fälle nur mit Hilfe der Balsteriologie aufgeklärt werden können. Die Frage wird noch weiter dadurch verwickelt, daß manchmal auch bei Pérsonen aus dèr Umgebung einer Epidemie Ruhrbazillen nachgewiesen werden können, die überhaupt nicht erkrankt sind oder bei denen vielleicht die Erkrankung so leicht verlief, daß sie nicht bemerkt wurde (Koch, Fraenkel, Rumpel, Kindberg).

Das Punctum saliens des ganzen Streites scheint mir in der rein praktischen Frage zu liegen, inwieweit ein gegebener Fall von Ruhr- oder ruhrühnlicher Erkrankung als ansteckungsgefährlich zu betrachten ist; dieselbe kann aber eben durch die bakteriologisehe Stuhluntersuchung nicht immer beantwortet werden.

Welchen Standpunkt soll nun der pathologische Anatom zu diesem Streit der Meinungen einnehmen? Soll er etwa bei einem tödlich verlaufenden Fall von "Colitis haemorrhagica" mit negativem bakteriologischen Befund, bei dem die Autopsie die für Ruhr charakteristischen Veränderungen auf den Tag brachte, die Diagnose „Dysenterie" ablehnen oder aber sich auf den Standpunkt stellen, daß , ,die Ruhr weder anatomiseh noch ätiologisch einen einheitlichen ProzeB darstellt" (Sternberg), und den Namen zwar beibehalten, den Begriff aber auch auf die supponierten, nichtspezifischen und nichtkontagiösen Prozesse erweitern, die mit ähnlichen anatomischen Veränderungen einhergehen sollen, oder schließlich den bakteriologischen und klinischen Befund unbeachtet lassën und überall dort, wo er die charakteristischen Darmveränderungen findet, auf der Diagnose „epidemische Ruhr" beharren (Adelheim)?

Hansemann, der die anatomische Differentialdiagnose der Ruhr am ausiührlichsten behandelt, scheint dieser Frage vorsichtig aus dem Wege gegangen zu sein, obzwar ihre eminente Wichtigkeit, von dem theoretisehen Interesse abgesehen, vor allem bei den gesundheitspolizeilichen Obduktionen einleuchtet.

Wie sind weiter die mehr oder weniger schweren, oft atypischen Darmaffektionen zu beurteilen, die nicht selten als Nebenbefund bei sonstigen schweren, zum Tode führenden Leiden aufgefunden werden und die sich klinisch entweder überhaupt nicht manifestierten oder vom Kliniker als Sekundärerscheinungen des Hauptleidens (urämischer, septischer Durchfall, sekundäre Darmtuberkulose usw.) aufgefaßt wurden? Diese und manche andere Fragen sind mir aufgetaucht, als ich in meiner Anstellung als. Prosektor am Reservespital Nr. 2 in Pardubitz (einer der größten militärischen Sanitätsanstalten in Böhmen) seit Sep- 
tember 1916 zahlreiche Fälle mit dysenterischen Darmveränderungen zu obduzieren hatte.

Deshalb bin ich an systematische bakteriologische Untersuchung des einschlägigen Sektionsmaterials herangetreten.

In der. Literatur ist nicht viel darïber zu finden. Die meisten Autoren begnügen sich damit, die anatomischen Befunde von Fällen wiederzugeben, die intra vitam bakteriologisch sichergestellt wurden. Nach Lentz können die Ruhrbazillen im Darminhalt, besonders in den Schleimflocken, in der geschwürig veränderten Darmwand und in den mesenterialen Lymphknoten von Ruhrleichen regelmäßig nachgewiesen werden. Im ganz entgegengesetzten Sinne äußern sich Gruber und Schaedel in einer vor kurzem publizierten Arbeit.

Seeligmann fand die Ruhrbazillen in 30 Prozent der obduzierten Fälle, Lampl in allen Fällen, wo ein für Ruhr typischer Befund vorlag, Ungermann und Jötten in zwei intra vitam erfolglos untersuchten Fällen.

Friedemann und Steinbock bekamen bei fünf Ruhrleichen ein nege.tives Resultat.

Systematische Untersuchungen an einem größeren Material sind meines Wissens noch nicht angestellt worden. Meine Untersuchungen umfassen etwa 100 Fälle und érstrecken sich auf ein Intervall von $1^{1} / 2$ Jahren. Die Zahl der von mir obduzierten Ruhrfälle war binnen dieser Zeit eine viel größere, ich konnte aber aus äußeren Ursachen nicht alle Fälle bakteriologisch untersichen. Doch war die Wahl der aur Untersuchung herangezogenen Fälle durch rein äußerliche Momente bedingt; höehstens war ich bestrebt, die anatomisch unklaren Fälle vorzuziehen.

Was aber meinem Material einen besonderen Wert für die Beurteilung der aufgeworfenen Fragen verleiht, ist weniger sein Umfang, als seine besondere Art: die Fälle stammen nämlich nicht von einer zeitlich und örtlich begrenzten Epidemie, sondern es handelt sich um Kranke, die von den verschiedensten Kriegsschauplätzen herkamen. Es liegt weiter in der Natur des Materials von einem Hinterlandspital, daß es sich bis auf kleine Ausnahmen um Fälle von längerer Dauer, zum Teil aber um ausgesprochen chronische Krankheitsformen handelte.

Die. Untersuchung geschah in der Weise, daB der herausgenommene und eröffnete Dickdarm mit Leitungswasser abgespült wurde, um möglichst alle Kotbestandteile zu entfernen ${ }^{1}$; dann wurde mit einer Platinöse ein Stïckchen eines Schorfes oder einer Pseudomembran entnommen und auf drei Endoplatten ausgestrichen. Wo keine Schorfe vorhanden waren,

1 Das hiesige Leitungswasser erwies sich bei wiederholter bakteriologischer Prüfung als einwandfrei. 
wurde etwas Schleim von der geröteten Schleimhautoberfläche oder vom Grunde der Geschwüre abgeschabt und in derselben Weise weiter bearbeitet. In einigen Fällen impfte ich auch von den submukösen Schleim. zysten oder Abszessen; das war immer bei den chronischen, in Abheilung übergehenden oder bereits vernarbten Prozessen der Fall, bei denen derartige Zysten oft massenhaft zu finden sind (Colitis cystica).

Stets wurden die frischen, mit Rötung und Schwellung der Schleimhaut einhergehenden Veränderungen bevorzugt, die bei chronischen Fällen neben den älteren, in Abklingen oder Abheilung begriffenen Prozessen fast immer vorkommen.

In den meisten Fällen wurden mehrere solche Plattenserien von verschiedenen Darmabschnitten angelegt. Dieses Yorgehen erwies sich als sehr zweckmäßig, da oft nur eine Plattenreihe Ruhrbazillen enthielt; in einigen Fällen wieder wurde ein Teil der Serien von Bact. proteus überwuchert, die übrigen dagegen blieben frei und ermöglichten das Abimpfen der verdächtigen Kolonien. Auf diese Weise wurden von jedem einzelnen Falle im ganzen 6 bis 12 Platten beimpft.

Nachdem die Arbeit Eisenbergs über eine Modifikation des Endoagars für Ruhrbazillenzüchtung erschienen war, habe ich dieselbe für meine Untersuchungen benutzt, $\mathrm{da}$ ich schon früher dieselbe Beobachtung wie Eisenberg sowie Schürer und Wolf gemacht habe, daß nämlich das freigewordene Fuchsin der Endoplatten, das von den Colikolonien in die Umgebung diffundiert, die Ruhrbazillen stark in ihrem Wachstum hemmt. Meine Erfahrungen mit den Eisenbergschen Nährboden waren sehr gut, obzwar sich auch bei ihm der tbelstand des Rotwerdens der Platten nicht völlig vermeiden läßt.

Nach etwa 20stündigem Bebrüten der Platten wurde eine Anzahd der verdächtig aussehenden Kolonien abgeimpft und in zickzackförmigen Strichen auf eine Lackmuslaktoseagarplatte übertragen. Von dieser wurden dann nach weiteren 24 Stunden die diagnostischen Nährböden beimpft und die Probeagglutination, eventuell auch die Austitrierung angestellt. Dieser Vorgang hat vor der üblichen Bearbeitung der Kolonien direkt von der Originalplatte nicht geringe Vorteile. Erstens scheiden diejenigen Kolonien, welche auf der Originalplatte eine zweifelhafte Reaktion zeigten, bei weiterem Prüfen sich aber als Milchzuckervergärer erweisen, durch ihr rotes Wachstum auf der Lackmusplatte von selbst aus; zweitens hat man reichliches Material zum Beimpfen der diagnostischen Nährböden zur Verfügung und kann auch die Austitrierung mit den spezifischen Sera von derselben Platte vornehmen; drittens entfallen manche Hemmungen bei der Probeagglutination, die sich beim Anstellen derselben von der Originalplatte oft in störender Weise bemerkbar machen. Außerdem kann man die Art des. Rasens auf dem dunkelblauen Nährboden viel besser als auf Schrägagar beurteilen. Ich habe oft die Beobachtung gemacht, daB manche auf der Originalplatte zart aussehenden Kolonien auf dem Lackmuslaktoseagar einen üppigen, opakweißen oder grauen Rasen bilden; es handelte sich dabei immer um sogenanntes weißes Coli oder Kokken, nie um pathogene Bakterien der Typhus-Ruhrgruppe, die sämtlich ein zartes, eigentümlich graublau durchschimmerndes Wachstum aufweisen, 
wobei die Differenzen zwischen den einzelnen Stämmen sehr gering sind und sich hauptsächlich nur in verschieden starker Blaufärbung des neutralen Nährbodens äußern. Man kann auf diese Weise die erstgenannten Kolonien von der weiteren Prüfung von vornherein ausschließen und sich viel nutzlose Mühe ersparen. Es kommen aber auch nichtpathogene Keime vor, denen dasselbe zarte Wachstum eigen ist, z. B. B. faecalis alcaligenes. Die Verzögerung des Resultates durch das Einschalten der zweiten Platte fiel natürlich bei meinen Untersuchungen nicht ins Gewicht. Zur direkten Aussaat habe ich die Conradischen Platten nicht benutzt, da mein Vorrat an Lackmuslösung sehr beschränkt war.

Die Probeagglutination wurde mit einer.Verdünnung $1: 100$, eventuell auch 1:250 geprüft, die endgültige Austitrierung entweder sofort ausgeschlossen oder erst nach einem längeren Zeitintervall, nachdem mehrere isolierte Stämme vorlagen, gruppenweise vorgenommen, und zwar ausschließlich im hängenden Tropfen, mit Ablesen unter dem Mikroskop. Zwar bält Lentz die makroskopische Methode bei der Rubrdiagnose für die einzig richtige, doch war ich durch die Knappheit der mir zur Verfügung stehenden Sera gezwungen, mich auf die mikroskopische Prüfung zu beschränken, was ich um so eher tun zu dürfen glaubte, als auch Kruse bei seinen umfangreichen Untersuchungen ausschließlich mit der mikroskopischen Methode gearbeitet hat; außerdem möchte ich hervorheben, daß man bei der mikroskopischen Prüfung der Agglutination viele interessante Details und Eigentümlichkeiten des Vorganges zu beobachten imstande ist, die natürlich bei der makroskopischen Methode der Beobachtung entgehen müssen. Auch kann man zugleich die Beweglichkeit sehr gut beurteilen (in der Kontrolle), hauptsächlich wenn man die Aufschwemmung mit Bouillon hergestellt hat.

Auf diese Weise habe ich in 52 von den 96 untersuchten Fällen Ruhrbazillen gefunden. Da aber in der Bakteriologie der Ruhr, hauptsächlich in bezug auf die Abgrenzung sowohl der einzelnen Rassen untereinander wie auch der Ruhrerreger im allgemeinen gegen ähnliche Bakierien; deren pathogene Bedeutung wenigstens zweifelhaft ist, noch mancher strittige Punkt übrig bleibt, halte ich es für nötig, die Kriterien an:zugeben, nach denen ich mich bei der Beurteilung der gefundenen Stämme und ihrer Zugehörigkeit zu den Ruhrbazillen richtete.

Daß ich nur diejenigen Stämme für Ruhrkulturen ansah, deren morphologische und tinktorielle Eigenschaften sowie das Wachstum auf gewöhnlichen Nährböden den Beschreibungen von Lentz, Kruse u.a. durchaus entsprach, versteht sich von selbst. Es sei nur hervorgehoben, daß meine sämtlichen Stämme unbeweglich waren, in Neutralrotagar mit Glykose keine Gasbildung und Fluoreszenz bewirkten und Glykosebouillon mit Lackmuszusatz röteten. Auf eine Besonderheit möehte ich aber etwas näher eingehen, da sie meines. Wissens bis jetzt unbeachtet geblieben ist. Auf Endoplatten, die direkt vom Infektionsmaterial beimpft waren, 
wuchsen die Ruhrbazillen in Form von zarten, mehr oder weniger durchsichtigen, 2 bis $3 \mathrm{~mm}$ breiten Kolonien mit etwas flachem, fein gekerbtem Saume; bei Lupenvergrößerung wies die ganze Kolonie, hauptsächlich aber der Randsaum, eine äußerst zarte, rippenartige Zeichnung auf.

Wenn man die Platte einige Tage bei Zimmertemperatur stehen läßt, so wird das Zentrum allmählich undurchsichtiger, manchmal leicht rötlich gefärbt, der Saum dagegen bleibt zart, breitet sich eher etwas aus, wobei seine Zeichnung noch auffallender wird.

Untersucht man das Material von einer solehen auch frisch geziichteten Kolonie im hängenden Tropfen, so sieht man es aus langen, ziemlich plumpen, unbeweglichen Fäden zusammengesetzt, so daß man an ihrer Zugehörigkeit zu der Typhus-Coligruppe zweifeln möchte. Bei der von der Originalplatte angestellten Probeagglutination ballen sich die Fäden zu bizarren Knäueln zusammen. Uberimpft man eine solche Kolonie auf Lackmuslaktose- oder Schrägagar, so ist man erstaunt, die Fäden mit einem Schlage zu den typischen, etwa zwischen dem Typhus und Coli stehenden Formen umgewandelt zu sehen.

In der zweiten Generation geht dieser Wachstumstypus meist verloren, die Kolonien sind dann auch auf Endoagar ziemlich glattrandig, ohne Zeichnung; nur einige Stämme vermochten die Eigentümlichkeit lange Zeit hindurch zu bewahren.

Ich fasse es als eine Involutionserscheinung auf, die durch den plötzliehen Mediumweehsel ausgelöst wird, wobei die Ruhrbazillen mit einem ihnen nicht besonders zusagenden Nährboden sich zu begnügen genötigt sind. In späteren Generationen tritt dann meist eine Gewöhnung an das künstliche Nährsubstrat ein. Ob die Erscheinung dem hohen Alkalitätsgrad oder dem Fuchsingehalt des Endo zugeschrieben werden soll, habe ich nicht näher untersucht; für die zweite Möglichkeit scheint mir aber der Umstand zu sprechen, daß sie auf der Eisenbergschen Modifikation in weit schwächerem Maße zum Vorschein kommt. Auf Lackmuslaktoseagàr habe ich sie nie beobachtet.

Ähnliche Wuchsformen beobachtete ich übrigens bei einigen Stämmen, die durch Agglutination nicht mit Sicherheit als Ruhrbazillen zu identjfizieren waren und auf die ich bei Bespreching der inagglutinablen Stämme weiter unten zurückkomme. Daß diese Eigenschaft der Ruhrbazillen meines Wissens bis jetzt nirgends erwähnt wurde, glaube ich dem Umstande zurechnen zu müssen, daß die meisten Untersucher bej Bearbeitung von Ruhrstühlen Lackmuslaktoseplatten benutzen. ${ }^{1}$

1 Ähnliche Wuchsformen bei Coli-, Typhus-, Ruhr-und anderen Bakterien der Typhus-Coligruppe haben Lingelsheim, Baerthlein, Gildemoister 
Die endgültige Bestimmung der jsolierten Stämme wurde der Prüfung mit hochspezifischem Immunserum vorbehalten.

Bokanntlich unterscheidet man mehrere Abarten von Ruhrbazillen, iiber deren gegenseitige Abgrenzung aber noch immer viel gestritten wird. Eine biologisch und scrologisch genügend scharf umschriebene Gruppe bildet der Shiga-Krusebazillus, welcher sich nach übereinstimmenden Angaben durch sein negatives Verhalten gegen Mannit, das Fehlen der Indolreaktion und die Fähigkeit, in flüssigen Nährmedien' lösliche Toxine zu bilden, von den übrigen Rassen unterscheidet; auch wird er durch ihre Immunsera nur in geringem Maße mitbeeinflußt. Ich habe Shiga-Krusebazillen nur in vicr Fällen gefunden; alle Stämme verhielten sich durehaus typisch. Ein weiterer Stamm (23), der bei der Probeagglutination mit Shiga-Kruseserum 1:100 reagierte, wurde leider vor der definitiven Prüfung irrtümlicherweise abseits gelegt; so daß ich mich über seinè Identität nicht mit Sicherheit aussprechen kann.

Viel weniger einheitlich sind die übrigen manitspaltenden Abarten, die von den amerikanischen Forschern ,Acidstämme“, von Kruse Pseudodysenteriebazillen genannt werden. Man kann unter ihnen mehrere Rassen unterscheiden, die sowohl serologisch wie auch mit Rücksicht auf die biologischen Merkmale untereinander abweichen. Es handelt sich vor allem um die von Flexner, Strong, Hiss und Russel (Y), Kruse und zuletzt Aronson gefundenen Abarten. Nach Lentz soll man die drei erstgenannten Typen 'durch ihr Verhalten gegenüber Maltose und Sacharose differenzieren können; auch andere Zuckerarten wurden von versehiedenen Autoren zu demselben Zwecke als brauchbar angegeben. Indessen hat sich aber gezeigt, daß die fermentativen Fähigkeiten der Bazillen auch bei einem und demselben Stamme ziemlich schwankend sind, hauptsächlich in bezug auf die Maltosevergärung. Deshalb verlangt Lentz, die Zuckerprüfung sofort nach der Isolierung aus dem menschlichen Körpor. zu untersuchen. Ảber auch damit ist nicht viel geholfen, denn eine Roihe von Arbsiten, darunter einige groß angelegte, haben gezeigt, daß das serologische Verhalten der Pseudoruhrstämme mit den

und Sonne beschrieben, doch handelte es sich bei den Beobachtungen von Lingelsheim und Sonne um alte Kulturen, wo sie ohne weiteres als Involutionsformen aufgefaßt werden können; die "Flatterformen" der Ruhrbazillen von Gildemeister verhielten sich in der Richtung von den oben beschriebenen different, daß sie als ,beständig umschlagende Sippen“ auch in den späteren Generationen neben Normalformen immer wieder vorkamen. Außerdem waren die abweichenden Kolonien bei Gildemeister und Sonne schwer oder nicht agglutinabel. Trotzdem glaube ich, daß es sich um verwandte Erscheinungen handelt. 
fermentativen Fähigkeiten nicht übereinstimmt (Ohno, Kruse und Mitarbeiter, Hutl, Sonne, Manteufel, Ebeling, Schroeter und Gutjahr, Winter, 'Keuper, Köhler, Schroeder und Umnus). Das darf um so weniger wundernehmen, als doch durch zahlreiche Arbeiten (Bernhardt, Ditthorn u. a.) bewiesen wurde, dab die biologischen Eigenschaften der Bakterien in gewissen Grenzen veränderlich sind, daß sie also keine konstanten Merkmale darstellen, auf Grund deren die Abgrenzung und Charakterisierung verschiedener Bakterienarten unter allen Umständen möglich wäre; es handelt sich vielmehr um ernährungsphysiologische und Stoffwechselvorgänge - Dinge, die in anderen naturwissenschaltlichen Disziplinen als Merkmale für die Abgrenzung von Arten nicht anerkannt werden (Loewenthal).

Aus diesem Grunde hat man versucht, atoxische Ruhrstämme auf Grund ihres serologischen Verhaltens zu -differenzieren.

Kruse, der sich mit seinen Schülern mit diesem Gegenstand am eingehendsten befaßte, unterscheidet im ganzen neun Rassen, die er mit den Buchstaben $A$ bis $J$ bezeichnet. Nun geht aber aus seinen Untersuchungen hervor, daß sich die einzolnen Abarten dareh einfache Agglutination mit hochwertigen Soris nicht unterseheiden lassen, da sie in hohem Grade gegenseitige Mitagglutination zeigen. Deshalb hat Kruse die Unterscheidung auf Grund des Absättigungsverfahrens (Castellanischen Versuches) durchgeführt. Seine Einteilung wurde zwar von vielen Seiten bekrittelt, ihre Riehtigkeit wird aber neuerdings von Schmitz bestätigt.

Für die praktisehen Zwecke ist sie allerdings zu kompliziert und in bozug auf den Castellanischen Versuch viel zu umständlich. Deshalb bin ich dem Vorschlag mancher Autoren gefolgt, auf nähere Differenzierung der Abarten überhaupt zu verzichten und sich einfach mit der Diagnose atoxische Ruhr oder Pseudodysenterie zufrieden zu stellen.

Die mir zur Verfügung stehenden Sera stammten zum Teil von den Sächsischen Sorumwerken, größtenteils aber vom Serotherapeutischen Institut in Wien. Es waren meist Kaninchensera und waren als Flexner und $\mathrm{Y}$ bezaichnet; der Titer betrug 2000 bis 4000 . Die Provenienz der Stämme, welehe zu ihrer Herstellung verwendet wurden, ist mir nicht bekannt. Da dieselben Sera auch bei den im hiesigen bakteriolugischen Laboratorium (Dr. Ren๕) durchgefuihrten Stuhluntersuchungen vom klinischen Material benutzt wurden, waren die einzelnen Serien meist rasch verbraucht, so daß ich bei meinen auf ein größeres Intervall sich erstreckenden Untersuchungen mit verschiedenen Seris eines jeden Stammes arbsiten mußte. Dabei zeigten sich nicht unbeträchtliche Differenzen zwischen den einzelnen Lieferungen in bezug auf ihr Verhalten den ver- 
schiedenen Rassen (Kontrollstämme) gegenüber; manche erwiesen sich als ziemlich spezifisch, andere dagegen agglutinierten die meisten Stämme in fast gleicher Verdünnung. Ein ähnliches Verhalten der Immunsera haben übrigens viele Untersucher beobachtet.

Da ich mir zwar die Vertreter der Kruseschen Hauptrassen vom Kralsehen Museum zukommen ließ, zur Herstellung von Immunsera aber kein genügendes Tiermaterial besa $\beta$, mußte ich leider auf die Differenzierung der gefundenen Stämme nach dem Kruseschen Schema verzichten. Ich begnügte mich also mit der Feststellung, da $B$ der in Frage stehende Stamm, bei sonstigem typischen Verhalten, durch ein oder mehrere der oben genannten Sera bis zu einer gewissen Titerhöhe agglutiniert wird.

Die meișten Stämme agglutinierten mit dem einen oder anderen Serum bis zur Titergrenze; bei einigen dagegen war eine positive. Agglutination nur etwa bis zu einem Viertel der Titerhöhe zu erreichen. Ich glaube, daß dies nicht gegen ihre Zugehörigkeit zu den Ruhrbazillen spricht, denn erstens kommen solche geringfügigen Schwankungen der Agglutinabilität der einzelnen Stämme bei verschiedensten pathogenen Bakterien vor - Krus e verzeichnet sie sogár innerhalb seiner Gruppen -, zweitens mußte ich aber damit rechnen, daß ich wahrseheinlich Vertreter von verschiedenen Rassen unter den Händen habe, die beobachtete Agglutination also eigentlich nur eine Mitagglutination ist, die natürlich die Titergrenze nicht immer erreichen muß. So wurden auch die mir zur Verfügung stehenden Vertreter der Kruseschen Rassen A, D, E und $\mathrm{H}$ von dem einen oder anderen der Sera wenigstens 1: 500 agglutiniert.

Das Verhalten den Kohlehydraten gegenüber wurde mit. Hilfe von Lackmusagarplatten mit 1 Prozent Zuckerzusatz nach Lentz geprüft; ich habe dieses Verfahren dem flüssigen Nähirboden von Hetsch und Doerr vorgezogen, weil ich auf derselben Platte mehrere Stämme zugleich durch kleine radiäre Striche untersuchen konnte, was einerseits nicht unerhebliche Materialersparnis bedeutete, andererseits aber direktes Vergleichen verschiedener Stämme ermöglichte.

Das serologische Verhalten der Stämme war mit dem fermentativen in vielen Fällen nicht in Utbereinstimmung. Nach dem oben Gesagten halte ich es aber nicht für nötig, die betreffenden Stämme deshalb als atypisch zu bezeichnen, um so weniger, als ich die Zuckerprüfung nicht immer gleich nach der Isolierung vornehmen konnte.

Dagegen halte ich diese Bozeichnung bei den zwei weiteren Stämmen (39 und 50) für berechtigt. Der erste wurde in zahlreichen Kolonien von typischer Wuchs.orm aus einem Falle von chroniseher Lungentuberkulose gezüchtet, bei dem als Nebenbefund eine rezidivierende Ruhr ohne tiefere 
Ulzerationen festgestellt wurde. Der Stamm spaltete keine von den drei Zuckerarten und verhielt sich auch sonst kulturell wie ein Shiga-Krusebazillus. Er wurde anfangs von dem Shiga-Kruseserum und von einem Y-Serum bis 1:500 agglutiniert, bei späterer Nachprüfung zeigte er aber ein schwankendes Verhalten, um schließlich nach 3 Monaten fast völlig die Agglutinabilität zu verlieren.

Der andere stammte von einer wahrscheinlich sehr alten Colitis cystica mit leichtem Rezidiv; er wurde aus einer Schleimzyste in wenigen Kolonien gezïchtet. Von einem Y-Serum wurde er bis 1:500 unvollständig agglutiniert und vermochte Mannit gar nicht, Maltose und Sacharose nur schwach zu säuern.

Die Klassifizierung dieser beiden Stämme ist schwierig. Für atypisch halte ich sie, falls es sich überhaupt um Ruhrbazillen handelt, deshalb, weil Mannit nicht angreifende Pseudoruhrstämme meines Wissens bis jetzt nicht bekannt sind, mit Ausnahme der später zu besprechenden Rasse J von Kruse und des Schmitzschen Bazillus, die aber mit den übrigen Rassen keine Mitagglutination zeigen. Nur bei Shiga und Amako finde ich eine Abart von Pseudoruhrbazillen erwähnt (Rasse V), die Mannit nur schwach säuern soll, und auch Manteufel hat in Ostafrika einen ähnlichen Stamm gefunden (Inder); im übrigen soll aber die Mannitvergärung, wenn man 1 prozentige Lösung verwendet, durchaus konstant sein (Kruse). Fuir Shiga-Krusebazillen sie zu halten, ist man natïlich nicht berechtigt, wegen des abweichenden Verhaltens im Agglutinationsversuch. Für die Ruhrerregernatur der beiden Stämme spricht neben dem sonstigen kulturellen Verhalten bei dem einen die große Zahl der gezüchteten Kolonien, bei dem zweiten der Umstand, daß er aus einer Schleimzyste gewonnen wurde. Ich halte dieselbe für wahrscheinlich; Beweise lassen sich natürlich nicht erbringen.

In einer weiteren Gruppe von acht Fällen (7., 8., 10., 11., 35., 42., 64., 69.) wurden Stämme gezüchtet, die sich kulturell and morphologisch von Ruhrbazillen nicht unterscheiden ließen, die aber keine nennenswerte Agglutination mit den Ruhrseris zeigten. Der eine stammte von einem Fall von chronischer rezidivierender Ruhr mit Ödemen, die übrigen von Fällen, welche mit mehr oder weniger vorgeschrittener Tuborkulose kombiniert waren.

Derartige ruhrähnliche; nichtagglutinable Stämme werden in der Literatur vielfach erwähnt. Sonne fand unter den von ihm untersuchten Pseudoruhrstämmen mehrere, die, untereinander verwandt, von den übrigen Pseudoruhrseris fast gar nicht mitagglutiniert wurden (Gruppe III); ihre Pathogenität hält er für bewiesen. Er äußert die 
Meinung, daß die von Doerr, Hilgermann, Morgan, Leiner, Böse, Lösener, Koponaris, Sangiorgi und Bangioannini beschriebenen inagglutinablen Ruhrbazillen mit den seinigen identisch seien. Die von ihm als Gruppe III aufgestellte Abart schließt aber auch die durch Baerthlein aus der Berliner Epidemie 1912 isolierten Stämme ein, die andererseits von Hutt nachgeprift und mit der Krusesehen Rasse E identifiziert worden sind. Schwer agglutinable Pseudoruhrstämme, die aber später volle Agglutinabilität wiedergewonnen haben, fanden weiterhin Keck und Keuper; auch bei Kruse, Sternberg und Salus finde ich sie erwähnt. Nach Gildemeister weisen manchmal frisch aus dem Stuhl gezüchtete Pseudoruhrbazillen eine abweichende Kolonienform auf (sogenannte Flatteriormen); diese Kolonien sollen schwer verklebbar sein, spalten aber bereits in der zweiten Generation typische Kolonien ab, die eine normale Agglutinabilität besitzen. Seeligmann fand Stämme, die mit Ausnahme der schweren Agglutinabilität wie Flexnerbazillen sich verhielten, in bezug auf die antigenen Eigenschaften teils mit diesen verwandt waren, teils aber eine Sonderstellung einnahmen; er faßt sie als Ubergänge von Saprophyten $\mathrm{zu}$,den echten Ruhrbazillen auf. Sehwer agglutinable Shiga-Krusestämme beschrieben Ungermann und Jötten, Seeligmann und Falta und Kohn. Hamburger und Bauch untersuchten eine. Rsihe von derartigen Stämmen, die sich in bezug auf mangelnde Mannitspaltung wie Shiga-Krusebazillen verhielten; ein Teil von ihnen erlangte die Agglutinabilität schon nach wiederholtem Uberimpfen, boi anderen war dies erst durch Anwendung gekochter Aufschwemmung nach Porges zu erzielen; die übrigen Stämme erwiesen sich schließlich auch bei Anwendung dieser Kunstgriffe als dauernd inagglutinabel. Bei einer späteren Nachprüfung konnte Bauch die letztgenannten Stämme mit der durch Sehmitz beschriébenen Ruhrbazillenabart identifizieren.

Der Schmitzsche Bazillus, der bei einer umfangreichen Epidemie in einem Gafangenenlager bei zahlreichen Fällen gezüchtet wurde, unterscheidet sich von dem Shiga-Kruseschen außer durch mangelnde Agglutinabilität mit den Ruhrseris hauptsächlich durch positive Indolreaktion. Nannit greift er nicht an, dagegen nach Lampl Sacharose. Kruse hält ihn für verwandt mit seiner Rasse J, die er bei einer Epidemie im Sanatorium Dösen isolierte und die sich ebenfalls in serologischer Hinsicht durch strenge Sonderstellung den übrigen Pseudoruhrrassen gegenüber auszeichnet. Ubrigens wird die pathogene Bedeutung des Schmitzschen. Bazillus angezweifelt (Gehrmann).

Meine diesbezüglichen Stämme waren zu der Zeit, wo die letztgenannten Arbeiten erschienen sind, leider zum größten Teil schon ab- 
gestorben, so daß eine Nachprüfung derselben nicht möglich war. Bei zwei von ihnen ( 35 und 64) wurde nach $15^{1 / 2}$ bzw, 14 Monaten ein gleichfalls negatives Verhalten im Agglutinationsversuch, allerdings nur den oben angeführten Seris gegenüber, konstatiert.

Obwohl bei einigen der von mir gefundenen inagglutinablen Stämme für die Zugehörigkeit zu den Ruhrbazillen bzw. für ihre ätiologische Beziehung zu der vorliegenden Darmaffektion manches zu sprechen scheint, so die typische Kolonienform $(42,64,69)$, der Spermageruch der Kulturen (69) sowie die große Zahl der gezüchteten Kolonien (42, 64), halte ich es - entgegen Sternberg - nicht für angängig, sie bloß auf 'Grund des kulturellen Verhaltens glattweg für Ruhrbazillen zu erklären. Nach Sonne, Salus, Egyedi und Kulka u. a. kommen nämlich in den Stühlen Bakterien vor, die sich kulturell ganz ähnlich wie Ruhrbazillen verhalten, mit denselben aber gar nichts 'Gemeinsames haben und zum Teil auch ganz differente Rezeptoren besitzen; besonders bemerkenswert scheint mir die Beobachtung von Salus zu sein, der bei drei solchen flexnerähnlichen Stämmen später Gasbildung im Traubenzucker fand. Ich komme noch weiter unten auf die Kriterien zu sprechen, die ich zur Aufstellung einer neuen Bazillenart als Krankheitserreger für erforderlich halte; die Frage nach der ätiologischen Bedeutung der fraglichen Stämme für die betreffenden Darmveränderungen glaube ich vorläufig offen lassen zu mïssen.

Indem also die serologische Prüfung trotz der zeitweise vorkommenden Abschwächung der Agglutinabilität einzelner Stämme nach allgemeiner Utberzeugung sozusagen den Schlußstein bei der Identifizierung eines pathogenen Bakteriums bildet, bedarf doch dieser Satz einer gewissen Einschränkung, und zwar in der Richtung, daß auch das kulturelle Verhalten nicht $z u$ vernachlässigen ist.

Es zeigte sich nämlich, daß bei frisch gezuichteten Stämmen von Typhus- und Ruhrbazillen eine wechselseitige Beeinflussung durch heterologe Immunsera vorkommt, die allerdings bei verschiedenen Seris verschieden stark ist (Gaehtgens). Ferner fand man mit Ruhrseris agglutinierte Bakterien, die in ihrem sonstigen Verhalten derartige Differenzen von Ruhrbazillen aufweisen, daß sie nicht ohne weiteres mit denselben zu identifizieren sind. Kuhn und Woithe, die als erste diese Beobachtung gemacht haben, züchteten aus dem Stuhle von einem Ruhrkranken neben Ruhrbazillen Coli und grampositive Kokken, deren sämtliche auf der Flatte vorhandenen Kolonien mit einem Flexnerserum in hoher Verdünnung agglutiniert wurden; auch das Serum des Patienten beeinflußte sie. Sie haben diese Erscheinung "Paragglutination" benannt und wollen 
sie damit erklären, daß bei dem Zusammenleben der heterologen Bakterien mit den Ruhrbazillen im Körper 'des Kranken den ersteren allmählich Rezeptoren für die Agglutinine des Serums angezïchtet werden. Die Lehre von der Paragglutination wurde dann durch eine Reihe von Arbeiten bestätigt und erweitert (Kuhn, Gildemeister und Woithe, Rimpan, Gildemeister und Baerthlein, Baerthlein, Kuhn, Ebeling, Sonne, Winter, Ditthorn und Neumark, Gieskiewitz, Busson, SachsMücke, Flatzek, Beitker sowie Schroeder und Umnus u. a.). Außer Colibazillen wurden auf Drigalski blau wachsende paragglutinable Stämme gefunden, die eher mit Ruhrbazillen zu verwechseln sind, von denen sie sich durch Gasbildung im Traubenzucker und Beweglichkeit unterscheiden (sogenannte Dahlemstämme von Gildemeister und Baerthlein). Baerthlein schreibt den paragglutinablen Bakterien auch eine praktische Bedeutung zu, und zwar in der Riehtung, daß sie als "Leitbakterien" bei der Suche nach pathogenen Keimen behilflich sein können.

Nach Paltauf soll dic Paragglutination durch Adsorption von fremden Rezeptoren zustande kommen. Die ursprüngliche Angabe Kuhn und Gildemeisters, daß die Paragglutination eine ziemlich rasch vorïbergehen de Erscheinung ist, die beim öfteren Utberimpfen der Stämme bald schwindet, hat zu Mißverständnissen Anlaß gegeben. Es zeigte sich, daß manche Stämme ihre Agglutinabilität recht lange behalten können, was manche Autoren veranlaßte, die Erscheinung nicht als Paragglutination aufzufassen (Flatzek, Gieskiewitz, Manteufel, Sachs-Mücke u. a.). In einer späteren Publikation sieht sich deshalb Kuhn gezwungen, den Begxiff der Paragglutination in etwas abgeänderter Weise zu präzisieren, indem er die Vergänglichkeit des Phänomens innerhalb einèr beschränkten Beobachtungszeit für nicht wesentlich erklärt.

Für die Hypothese von 'Kuhn und Woithe spricht unter anderem auch die künstliche Erzeugung der Paragglutination, die nach mehreren fehlgeschlagenen Versuchen (Kuhn; Woithe und Gildemeister, Rimpan, Markoff) schlieBlich doch gelungen ist, und zwar durch Kultivierung von Colibazillen auf 'Nähragar, der mit Bouillonkulturen des pathogenen Stammes hergestellt war (Kuhn und Ebeling).

Wenn also für manche Fälle heterologer Agglutination die Auffassung derselben als Paragglutination richtig sein mag, so gibt es doch eine Reihe von Beobachtungen, welche auf diesem Wege nicht restlos erklärt werden können. Es wurden nämlich in Stühlen von Kranken Stämme gefunden, die mit dem Immunserum des in Betracht kommenden pathogenen Bakteriums nicht oder nur schwach, mit anderen Seris dagegen hoch 
agglutinierten (Dithorn und Neumark, Rimpan, Sachs-Mücke). Weiterhin fand man coliähnliche, mit Ruhrserum agglutinierende Bakterien auch bei Gesunden (Egyedi) und außerhalb des menschlichen 'Körpers (Winter, Busson). Egyedi konnte derartige Stämme mit Regelmäßigkeit aus Pferdemist ziichten; er faßt sie als obligate Saprophyten des Pferdeserums auf, wodurch vielleicht ihre Agglutinabilität im NormalPferdeserum zu erklären sei. Von hier können sie mit Leichtigkeit in den menschlichen Darm gelangen. Sie werden durch verschiedene Kaninchen- und Plerdeimmunsera agglutinjert, aber in einer ganz regellosen Weise, die auf eine. Spezifität des Phänomens nicht schließen läßt; Egyedi nennt diese Erscheinung ,paradoxe Agglutination". Auf Grund dieser Beobachtung spricht er den agglutinablen Nichtruhrstämmen jede Bedeutung für die Diagnose ab. Da diese Bakterien außerdem noch sehr bunte kulturelle Eigenschaften besitzen, ja manchmal ein den Ruhrbazillen sehr ähnliches Verhalten aufweisen (blaues Wachstum auf Drigalski, fehlende Gasbildung in Traubenzuckeragar und mangelnde Beweglichkeit), so können sie, namentlich beim flüchtigen Arbeiten, zu diagnostischen Irrtümern AnlaB geben.

Aus dem bis jetzt Gesagten geht hervor, daß die Agglutination im spezifischen Immunserum allein zur Identifizierung eines Bakteriums nicht genügt; nicht einmal durch den Absättigungsversuch kann hier Abhilfe geschaffen werden,' da im Gegensatz zu der Mitagglutination der Castelanische Versuch sowohl bei der Kuhnschen Paragglutination wie auch oft bei der Egyedischen ,paradoxen Agglutination“ positi ausfällt (Egyedi, Flatzek).

Nun gibt es aber auch eine andere Auffassung der Frage, die nicht kurzweg von der Hand zu weisen ist. Wenn einerseits gewisse Schwankungen im bjologischen Verhalten der Ruhrbazillen (Maltosespaltung) anerkannt werden, so ist es prinzipiell nicht einzusehen, warum das Fehlen der Gasbildung in Traubenzucker, deren Unbeständigkeit doch bei anderen Bakterien (Coli, Paratyphus) bekannt ist, gerade bei Ruhrbazillen ein artspezifisches Merkmal sein müßte, dem zu willen man die Spezifität der Agglutination anzweifeln sollte. So fassen O. Mayer, Manteufel, Falta und Kohn ihre gasbildenden agglutinablen Stämme als atypische Ruhrbazillen bzw. als Utbergangsstufen zwischen Coli- und Ruhrbazillen auf. Einige experimentelle Beobachtungen scheinen für diese Auffassung zu sprechen; O. Mayer fand bei Gesunden aus der Umgebung der Fürther Epidemie zwei gasbildende flexneragglutinable Stämme, die sich nach der Mäuscpassage wie Pseudoruhrbazillen verhielten. Falta und Kohn ist es gelungen, die 'Gasbildung ihrer agglutinablen Stämme 
sowohl durch Tierversuch wie auch in Kulturen in beiden Richtungen zu beeinflussen. Mayer läßt aber selbst die Möglichkeit zu, daß er bei seinem Versuch von einer Mischkultur ausgegangen sei; seine durch den Tierversuch abgeänderten. Stämme zeigten nicht unbeträchtliche Abweichungen von der Ausgangskultur im serologischen Verhalten (Hutt) und bei späteren Versuchen gelang es ihm nicht mehr, den gasbildenden Stamm durch 'Tierpassage irgendwie abzuändern. Die Befunde von Manteufel, Falta und Kohn hält Kuhn für paragglutinable Coli; gerade die aus den pathologischen Stühlen herausgezüchteten Colistämme sollen sich oft durch beträchtliche Sehwankungen des Gärungsvermögens auszeichnen.

Ich hatte bei meinen Untersuchungen wenig Gelegenheit gehabt, mich mit den agglutinablen Gasbildnern zu befassen, was dem Umstande zuzurechnen ist, daß ich das Verhalten im Traubenzuckeragar meist vor dèr Agglutination geprüft habe, Eine einzige Ausnahme bildet der Fall 34, bei welchem zahlreiche Kolonien, von einem unbeweglichen Bakterium wuchsen, das bei der Probeagglutination mit einem Y-Serum 1:50 reagierte, in Neutralrotglykoxagar aber Gasbildung und starke Reduktion bewirkte; es wurde weiter nicht untersucht. Das Ziel, nach welchem ich bei meinen Untersuchungen strebte, bestand, wie anfangs erwähnt, in erster Linie darin, über die Zugehörigkeit der vorliegenden Darmaffektionen $\mathrm{zu}$ der epidemischen Ruhr ins klare zu kommen; zu diesem $Z$ wecke hieß es vor allem nach denjenigen Bakterien zu fahnden, die als Ruhrerreger allgemein anerkannt sind und deren pathogene Bedeutung einerseits durch ihr regelmäßiges Vorkommen bei der Ruhr, andererseits durch den positiven Ausfall des Tierversuches (am Affen, Bernhard und Merkoff) wie auch durch Menscheninfektionen mit Reinkulturen, teils absichtliche (Strong, Jehle), teils zufällige (Kruse, Karlinski, Heuser), für bewiesen gelten muß. Damit soll natürlich nicht in Abrede gestellt werden, daß möglicherweise auch andere Bakterien das klinische und anatomische Bild der Ruhr erzeugen können. So wurden außer den oben besprochenen agglutinablen Gasbildnern auch andere, mit Ruhrseris nicht agglutinierende, mehr oder weniger paratyphusähnliche Bakterien (sogenannte Paradysenterie Kruse) vielfach als Ruhrerreger angesprochen (Jehle, Leiner, Martini, Lösener, Loewen thal, Deycke und Reschad, Rimpan). Schließlich ist auch die von französischen und italienischen Autoren (Rodet und Layriffont) vertretene, neuerdings von Marx, Köhlisch, Menzer, Seeligmann u, a. wieder aufgenommene Ansicht nicht von der Hand zu weisen, daß möglicherweise die Ruhr- und andere pathogene Bakterien im mensch- 
lichen Organismus durch allmähliche Umwandlung der Colibazillen entstehen. Besonders verlockend für die Untersuchungen am pathologischanatomischen Material muß der Vorschlag von Köhlisch erscheinen, im kranken Organismus sozusagen botanisieren zu gehen; $d$. h. auf die verschiedenen Varietäten des Colibazillus die Aufmerksamkeit zu lenken, welche durch Verlust des Gärvermögens und zuweilen auch der Beweglichkeit, zum Teil auch durch Abänderung des Kolonientypus sich den Ruhrbazillen in kultureller Hinsicht mehr oder weniger annähern und bei der supponierten Entstehung der pathogenen Keime die Ubergangsstufen vorstellen sollen. Daß in den pathologischen Stiihlen oft Bakterien zu finden sind, die in bezug auf Laktose- und Glykosevergärung und Beweglichkeit vom typischen Coli mehr oder weniger abweichen, so daß sich aus ihnen eine kontinuierliche Reihe zusammenstellen läßt, die anscheinend einen fließenden Ubergang zu den pathogenen Bakterien bildet, ist seit langem bekannt (Gräf, Kuhn, Gildemeister und Baerthlein, Salus). Auch zeichnen sich derartige Stämme oft durch Verändérlichkeit ihrer kulturellen Eigenschaften aus; manchmal lassen sich dabei mutationsartige Vorgänge beobachten (Loewenthal, Sachs-Mücke, Salus). Eine andere Frage ist es aber, wie man solche Befunde im Einzelfalle bewerten soll. Hat man einen derartigen "atypisehen" Stamm fast in Reinkultur direkt aus den krankhaften Produkten der Darmwand herausgezüchtet, so kann man natürlich mit einer gewissen Wahrscheinlichkeit annehmen, daB er zu dem Krankheitsprozeß in ätiologischer Beziehung steht. Bewiesen ist es aber damit nicht, und die' Geschichte der Bakteriologie liefert dor Beispiele genug, wie man auf diese Weise auf falsche Fährte geraten kann; ich will nur auf die Rolle der Salmonschen Bazillen bei. der Schweinepest hinweisen. Auch die Agglutination des fraglichen Bakteriums in Krankenserum ist in dieser Hinsicht nicht mit Sicherheit zu verwerten, da auch 'die paragglutinablen Stämme durch dasselbe oft stark beeinflußt werden (Baerthlein). Sehließlich wäre an das-Heranziehen' des Tierexperimentes zu denken; aber bei den kleinen Laboratoriumstieren läßt sich bekanntlich nicht einmal durch echte Ruhrbazillen ein dem menschlichen ähnliches 'Krankheitsbild erzeugen, und mit dem Nachweis der Giftwirkung ist nicht viel besagt, da auch die Dahlemstänme von Gildemeister und Baerthlein oft beträchtliche Giftigkeit besitzen. Andererseits wird von Kruse betont, dab bei seinen Stämmen trotz langjähriger Beobachtung und wiederholter Prüfung derartige Veränderungen im kulturellen Verhalten nie festgestellt werden konnten, welche auf einen Rückschlag zum Colitypus hindeuten würden. 
Aus diesen Gründen habe ich davon Abstand genommen, mit den gasbildenden Stämmen, von denen sehr oft einige Kolonien neben typischen Coli auf den Platten vorhanden waren, mich näher zu befassen. Andererseits glaube ich, mit genügend scharfer Kritik vorgegangen zu sein, um Sicherheit zu haben, daß die von mir als solche angesprochenen Stämme wirkliche Dysenteriebazillen waren.

Etwas anders verhält es sich mit der Frage nach der ätiologischen Rolle der Paratyphusbazillen für das Zustandekommen ruhrartiger Erkrankungen. Außer der typhösen und gastroenterischen Form sollen nämlich beì der Paratyphusinfektion Fälle vorkommen, die mit geschwürigen Prozassen im Dickdarm einhergehen, welche anatomisch von der Ruhr nicht zu unterscheiden sind. Lucksch, Scott und Wells sowie Herford bezaichnen die bei ihren Paratyphusfällen vorgefundenen Geschwüre als dysenterieartig, und Korczynski, Herxheimer, Stephan und Galambos haben bei Paratyphusfällen Befunde crhoben, die mit gewöhnlicher Ruhr völlig überreinstimmen. Dagegen fassen Helly und Sternberg derartige Befunde als Mischinfektionen auf, wenn es sich nicht um Fälle handelt, bei welchen, wie das oft der Fall ist, die Diagnose nur auf Grund des bekanntlich nur mit Vorsicht verwertbaren Befundes von. Paratyphusbazillen im Stuhl intra vitam gestellt worden war. Andererseits kann aber auch die enteritische Form von Paratyphus, welche anatomisch meist als Enteritis follicularis sich kennzeichnet, mit blutigschleimigen Stühlen einhergehen, ohne daß tiefere, für Ruhr charakteristische Veränderungen vorhanden sein müßten (Sternberg). Unsicher ist auch die ätiologische Beziehung der von Neukirch aus dem Krankenblute gezüchteten Bazillen der Glässer-Voldagsengruppe (B. Erdzindjan) $z u$ den dysenterieartigen Veränderungen, die bei einem Teil seiner Fälle das Krankheitsbild beherrschten.

Ich habe in keinem der hier besprochenen Fälle Paratyphusbazillen gefunden, obzwar ich sämtliche in dieser Richtung verdächtige Stämme serologisch prüfte.

Dagegen habe ich in zwei anderen, hier nicht näher zu besprechenden Fällen Paratyphus-B-Bazillen gefunden; bei einem handelte es sich um akute Gastroenteritis (Darminhalt und Galle), bei dem anderen war der Tod durch einen Schilddrüsenabszeß verursacht, in dem sich ParatyphusB-Bazillen in Rinkultur fanden; Darmveränderungen waren nicht vorhanden. Bei einem dritten Falle, der klinisch als fieberhafter Ikterus verlief, wurde anatomisch eine akute Cholangitis festgestellt, und aus der Galle wuchs Reinkultur von Paratyphus-A-Bazillen.

Zum Schlusse wäre noch das Vorkommen von Ruhrbazillen außerhalb des Darmtraktus kurz zu streifen. Bekanntlich pflegen die Ruhr- 
bazillen jhre pathogene Wirkung in der Darmwand zu entfalten, ohne in den Kreislauf uiberzutreten; höchstens gelangen sie bei akuten Fällen in die mesenterialen Lymphknoten. Doch gibt.es auch Ausnahmen von dieser Regel; so wurden von einer Reihe von Untersuchern teils intra vitam im kreisenden Blut, teils post mortem in Milz, Galle und Herzblut Ruhrbazillen gefunden (Markwald, Duval und Basett, Rosenthal, Knox und Schorer, Aveline, Boycott und Macdonald, v. Loghem, Mühlmann, Brückner, Fraenkel, Friedrich, Ghon und Roman, Nowicki, Seligmann, Ungermann und Jötten).

Ich habe keine systematische Untersuchungen in dieser Richtung angestellt; immerhin habe ich bei einigen Fällen Galle und Milz untersucht, letztere hauptsächlich dann, wenn sie vergrößert war. Ruhrbazillen konnte ich in keinem Falle finden.

Nebən dem direkten Nachweis des Krankheitserregers wird der Gehalt des Krankenserums an spezifischen Agglutininen für die Diagnose vieler Infektionskrankheiten benutzt. Utber den Wert der Agglutination mit Krankenseris bei Ruhr liegt eine stattliche Literatur vor, auf die ich nicht näher eingehen kann. Aus den zahlreichen Untersuchungen geht hervor, daß bei der Infektion mit Shiga-Krusebazillen der Agglutination ein gewisser diagnostischer Wert zukommt; bei der Pseudoruhr weichen dagegen die Urteile iiber die Spezifität der Reaktion weit voneinander ab. Von einer Anzahl Autoren wurden ziemlich hohe Agglutinationswerte bei Gesunden beobachtet; Keck konnte bei Darmstörungen verschiedener Art, wie z. B. Durchfälle bei Lungenphthise, eine deutliche unspezifische Steigerung der Agglutination feststellen. Dagegen soll nach Friedemann und Dünner auch hier die Agglutination spezifisch sein, wenn nur die grobflockige Reaktion beriicksichtigt wird. Die für Diagnosestellung erforderliche Titerhöhe wird zwischen 1:100 und 1:300 angegeben.

Boim Laichenmaterial war von vornherein nicht . viel von der Agglutination zu erwarten; es gilt nämlich als allgemein anerkannte Tatsache, daß bei tödlich verlaufenden Infektionen, hauptsächlich wenn sie mit schwerem kachektischen Zustand einhergehen, die Bildung von Antikörpern darniederzuliegen pflegt. So konnten Vaillar'd und Dopter und Rosenthal bei Fällen mit letalem Ausgang keine Agglutination feststellen, und auch Shiga fand in den letal verlaufenden Fällen sehr sehwache Agglutination. Dagegen haben Gruber und Schaedel nach einer kürzlich erschienenen Mitteilung in 9 Fällen von autoptisch festgestellter Dysenterie positive Agglutination mit Leichenserum bekommen und schreiben dieser Reaktion einen hohen Wert für die Aufklärung der Ätiologie bei fraglichen Darmaffektionen zu. 
Ich versuchte an Anfang meiner Untersuchungen, die Agglutination mit Leichenserum mir dienstlich zu machen, ließ aber im weiteren davon ab, hauptsächlich aus dem Grunde, weil die mir zuir Verfügung stehenden Kontrollkulturen - es handelte sich um alte Laboratoriumsstämme eine sehr leichte und dabei ungleichmäßige Verklebbarkeit aufwiesen, so daß die als spezifisch zu betrachtende Titerhöhe schwer zu ermitteln war. Nur in einem Falle (6) von viermonatiger Krankheitsdauer konnte ich eine ziemlich hohe Agglutination der Flexnerbazillen feststellen $(1: 640)$, die vielleicht als spezifisch anzusehen ist; Ruhrbazillen konnte ich bej diesem Fall im Darm, der in Abheilung begriffene Ruhrgeschwüre enthielt, nicht nachweisen.

Die anatomischen Befunde der untersuchten Fälle boten in bezug auf Darmveränderungen das bekannte äußerst wechselvolle Bild; zwischen den leichtesten mit Schwellung und Rötung der Schleimhaut und katarrhalischer Exsudation einhergehenden und den schwersten durch Zerstörung eines großen Teiles der Schleimhaut sich auszeichnenden Fällen ließen sich alle Ubergănge als kontinuierliche Reihe zusammenstellen. Akute Veränderungen waren, wie eingangs erwähnt, in der Minderzahl; bsi den meisten Fällen handelte es sich um spätere Stadien der Erkrankung, zum großen Teil aber um eminent chronische Formen, mit ihrem mannigfaltigen Bilde, das aus den Schilderungen der Autoren, von denen ich nur Orth, Heubner und Dopter nenne, genügend bekannt ist. Loider war es nicht in allen Fällen möglich, die Dauer der Erkrankung festzustellen; die mir zur Verfügung stehenden klinischen Angaben in den Vormerkblättern, soweit diese iiberhaupt noch zu erhalten waren, ließen in bezug auf Vollkommenheit und Genauigkeit viel zu wünschen übrig, wie das bei dem Massenbetrieb der 'Kriegszoiten nicht anders zu erwarten war. In vielen Fällen fand ich überhaupt keine Angaben über irgendwelche Symptome seitens des Darmtraktus; dies war hauptsächlich dann oft der Fall, wenn die Darmveränderungen neben einer anderen Erkrankung, die an sich zum Tode geführt hatte, als Nebenbefund erhoben wurden, nicht selten aber auch bei Fällen von chronischer Ruhr ohne anderweitige Komplikationen. Das kann nicht wundernehmen, denn es ist allgemein bokannt, wie wenig charakteristische Symptome die chronische Ruhr oft darbietet. Die kürzeste feststellbare Krankheitsdauer war 18 Tage (Fall 92). In Fällen, wo keine diesbezüglichen Angaben vorhanden waren, war ich darauf angewiesen, das Alter der Affektion nach dem vorliegenden anatomischen Befund annähernd zu schätzen. Das ist aber keine leichte 
Aufgabe, denn wie Löhlein mit Recht hervorhebt, lassen sich bei der Ruhr nur in den frischen Stadien der Erkrankung, bis zu etwa 3 Wochen, aus dem Charakter der Veränderungen gewisse Schlïsse auf das Alter des Falles ziehen; bei älteren Fällen dagegen, wo bereits das wechselvolle Bild der chronischen Ruhr vorliegt, mit dem Nebeneinander von älteren abheilenden Veränderungen und frischen Rezidiven bzw. Exazerbationen des Prozesses, ist ein derartiges Raten völlig unnütz, da es meist fehlschlägt. Aber nicht einmal die mich am meisten interessierende Frage, ob es sich um eine akute oder chronische Erkrankung handelt, ließ sich immer mit Sicherheit entscheiden. Wo tiefe Sshleimzysten, pigmentierte Narbən oder gereinigte Gaschwüre mit Zichen der Abheilung neben frischen diphtheritischen oder katarrhalischen Veränderungen vorliegen, da ist natürlich an einer längeren Dauer bzw. Chronizität des Prozesses nicht zu zweifeln; es kann aber vorkommen, dab bei einem sehweren Razidiv die frischen diphtheritischen Veränderungen sich nicht nur auf den früher verschont gebliebenen Partien, sondern auch auf der neugebildeten, die ehemaligen Geschwüre auskleidenden Schleimhaut etablieren, so daß die Spuren der ursprünglichen Erkrankung völlig verdeckt werden. Nicht einmal die mikroskopische Untersuchung führt hier immer zum Ziele, denn die neugebildete Schleimhaut kann, soweit es sich um flache, oberhalb der Muscularis mucosae liegende Geschwïre gehandelt hatte, nach kurzer Zeit ein völlig normales Aussehen erreichen (Beitzke). Aber auch bei einer katarrhalischen Affektion des Dickdarmes findet man manchmal geringe Pigmentierungen oder Narben im Sigma, die vielleicht die einzigen Uberreste einer früher überstandenen $\mathrm{Ruhr}$ darstellen; die gegenwärtige frische Erkrankung wäre somit als leichtes Rezidiv aufzufassen. Ich verfüge über eine Serie von Fällen (mit positivem Bazillenbefund), wo ich auf'Grund des autoptischen Befundes auf eine akute Erkrankung schließen muBte, obzwar die Patienten seit einer geraumen Zoit wegen eines anderen Loidens in Spitalbehandlung sich befanden. Zum Teil handelte es sich um ganz geringe katarrhalische Veränderungen, die als Nebenbefund zu deuten waren, zum Teil aber auch um ausgedehnte, schwere Erkrankungen, die an sich zum Tode führen mußten. Boi diesen Fällen wäre eine Klärung in betreff auf das Alter der Darmaffektion erwünscht gewesen, denn falls es sich nicht um Rezidive eines unbamerkt gebliebenen älteren Prozesses handelte, waren sie nicht anders als durch Spitalsinfektion zu erklären. Mir scheint übrigens diese Annahme nicht unwahrscheinlich, denn nieht erkannte Ruhrfälle waren, wie die Autopsien zeigten, auf zahlreichen Abteilungen des Spitals vorhanden und Ansteckungen von Pilegepersonal kamen auf den nicht für Rubrfälle 
bestimmten Baracken öfters vor. Das war um so leichter möglich, als manche Ärzte, wie ich mich beim Durchlesen der Vormerkblätter überzeugte, diejenigen Fälle von blutig-schleimigem Durchfall, bei denen die bakteriologische Untersuchung ein negatives Resultat ergab, einfach als Enteritis bezeichneten und von den für Ruhr nötigen Vorsichtsmaßregeln Abstand nahmen; erst bei der Autopsie zeigte sich dann der dysenterische Charakter der Erkrankung, der noch durch den Nachweis von Ruhrbazillen erhärtet wurde. Inzwischen figurierten aber die Fälle mit der falschen Diagnose und kamen auf nichtinfektiöse Baracken zu liegen, die dann natürlich leicht verseucht werden konnten.

Einige von diesen Fällen waren dadurch bomerkenswert, daß sie höchstwahrscheinlich die leichtesten Formen der Ruhr darstellen. Nach der allgemein gültigen Auffassung zeichnet sich das allererste Stadium der Ruhr durch katarrhalische Veränderungen im Darm aus; die Schleimhaut zeigt außer Rötung und Schwellung, vermehrter Schleimproduktion und vielleicht schon einigen Blutungen keine tieferen, für die späteren Stadien der Ruhr so charakteristischen Veränderungen. Natürlich kann auch in diesem Stadium unter schweren Intoxikationserscheinungen der Tod eintreten; das scheint hauptsächlich bei kleinen Kindern oft der Fall u sein, wie eine interessante Beobachtung von Keuper zeigt. In den weitaus meisten Fällen geht aber die Erkrankung nach Ablauf dieser Erscheinungen in Heilung über, oder aber es gesellen sich alsbald tiefere Veränderungen dazu, wie eiterige und fibrinöse Exsudationen auf die Oberfläche der Schleimhaut und in diese selbst sowie mehr oder weniger ausgedehnte Koagulationsnekrose, die zusammen das meist bei Autopsien vorgefundene Bild der Darmdiphtherie (im anatomischen Sinne) ansmachen. Da die Erkrankung von unten nach oben sich ausbreitet, so findet man meistens im unteren Abschnitt des Dickdarms die schwersten Veränderungen; nach oben nehmen diese allmählich an Intensität ab, bis man schließlich am obersten Rande der Affektion, oft erst jm Ileum, auf cine Partie kommt, die nur katarrhalische Veränderungen aufweist, ein Beweis, daß dieselben den übrigen vorausgehen. Die leichtesten Erkrankungen aber, die nur mit katarrhalischen Erscheinungen seitens des Darmes einhergehen, wie sie fast bei jeder Epidemie neben den schweren Formen, oft in großer Zahl, beobachtet werden, gehen in der Regel rasch in Heilung über und kommen daher nur äußerst selten dem pathologisehen Anatomen $z u$ Gesicht. Nur wenn die Ruhr Individuen befällt. die durch eine andere schwere Erkrankung oder aus sonst welchem Grunde schon vorher stark herabgekommen waren: kann diese an sich leichte Komplikation den Tod herbeiführen oder beschleunigen, und man hat dann 
Gelegenheit, sie auf dem Obduktionstisch zu untersuchen. Derartige Fälle hat Beitzke beschrieben. Ich glaube einige von meinen Fällen auf dieselbe Weise auffassen zu müssen.

Das trifft vor allem für den Fall $55 \mathrm{zu}$, bei dem zwar klinische Angaben fehlen, aber die vorgefundene schwere, ausgedehnte, käsige Tuberkulose des Urogenitalsystems den Schluß auf eine ziemlich lange Krankheitsdauer zuläßt; die Veränderungen im Darm beschränken sich auf eine Rötung der Schleimhaut, die zugleich eine etwas lederartige Beschaffenheit aufwies, was wahrscheinlich auf entzündliche Infiltration zurückzuführen ist; Geschwüre waren nirgends vorhanden, die Kontinuität der Schlejmhaut war völlig unversehrt; nur im Sigma waren einige pigmentierte Stellen zu finden, die aber zu chaiakteristisch waren, als daB man sie für Spuren einer abgelaufenen Ruhr ansprechen könnte. Weiter gehören die Fälle 57, 60, 67, 70, 71 hierher, bei denen es sich um eine Komplikation mit mehr oder weniger vorgeschrittener Lungentuberkulose handelte; einer von ihnen (57), bei dem leider auch nähere klinische Angaben fehlen, hatte außerdem eine Schußwunde, die das rechte Hüftenbein traf und aus der sich durch hämatogene Infektion tuberkulöse Ostitis entwickelte. Sämtliche fünf Fälle wiesen im Dickdarm oder Ileum Geschwüre auf, deren tuberkulöser Charakter unverkennlich war; nur bei zwei $(70,71)$ war daneben eine diffuse, die ganze Länge des Dickdarms betreffende katarrhalische Entzündung festzustellen, die übrigen wiesen viel geringere Veränderungen in bezug auf Intensität und Ausdehnung auf, so daB man in Verlegenheit war, ob sie überhaupt als Ruhr anzusehen sind. In den Lehrbüchern findet man die Angabe, daß bei ausgedehnter Darmtuberkulose die unterbalb der Geschwürsflächen liegende Schleimhaut oft katarrhalische bis pseudomembranöse Entzündung aufweist, die auf die Wirkung der aus der Wundfläche sezernierten Toxine zurückzuführen seien. Der einwandfreie Nachweis von Rubrbazillen ließ an der Zugehörigkeit der fraglichen Affektion zur Ruhr keine Zweifel bestehen.

$\mathrm{Ob}$ es sich aber bei sämtlichen diesen Fällen un akute, dann natürlich nicht anders als durch Spitalsinfektion zu erklärende Erkrankungen handelte, kann man auf Grund des autoptischen Befundes, wie aus dem oben Gesagten hervorgeht, nicht mit Sicherheit entscheiden.

Bei dem Falle 57 war neben den. Veränderungen im untersten Dickdarmabschnitt, die denen beim Fall 55 sehr ähnlich sahen, noch eine etwa $50 \mathrm{~cm}$ lange Partie des Ileum ergriffen; hier war die Schleimhaut gerötet, mit Blutungen durchsetzt und auf der Höhe der Querfalten, die wahrscheinlich infolge entzündlicher Schwellung der Schleimhaut sich gebildet hatten, oberflächliche Schorfe; der dazwischen liegende Darmabschnitt war frei von Veränderungen. Sowohl aus der Rectumschleimhaut wie auch aus den Schorfen im Ileum ließen sich Rubrbazillen züchten. Derartige getrennte Lokalisation des Ruhraffekts, die zudem mit der anatomischen Disposition offenbar nichts zu tun hat, läßt kaum eine andere Erklärung zu als diejenige, daß es sich ursprünglich um eine 
diffuse, bis in das Ileum reichende Erkrankung gehandelt haben mub, die zum größten Teil wieder restlos abheilte; nur in der zuletzt erkrankten Darmpartie (lleum) und des weiteren im Rectum, wo die Veränderungen am schwersten zu sein pflegen, haben sich noch Uberreste des krankhaften Prozesses erhalten. Leider waren von diesem Fall keine klinischen Aufzeichnungen zu bekommen. Bei den übrigen Fällen finden sich in den Vormerkblättern keine Angaben über etwaige Symptome seitens des Darmtraktus; nur im Fall 71 wird 5 Tage vor dem Tode über blutigschleimigen Durchfall berichtet. Weiter gehört höchstwahrscheinlich auch der Fall 54 hierher; hier sind aber keine genaueren Aufzeichnungen über den Darmbefund gemacht worden.

Bemerkenswert ist weitẹr Fall 53. Der Mann erkrankte im November 1917 an Malaria; die Anfälle dauerten bis 6. IX. Später entwickelten sich Odeme und klinisch nachweisbare Leberschwellung. Zuletzt schloß sich Albuminurie an und der Tod trat am 5. XII. unter urämischen Symptomen ein. Die Autopsie brachte eine Uberraschung; es wurde neben Spuren einer abgelaufenen Malaria (Melanose der Milz) ein Lymphosarkom des linken Lungenhilus gefunden, das in die Lunge hineinwuchs und in der Leber zahlreiche Metastasen bildete; außerdem bestand eine akute Glomerulonephritis. Im Rectum und Sigma fanden sich rötlichgraue, mit Blutungen durchsetzte Verschorfungen der Schleimhaut; dazwischen war nur eine dunkelgraue Pigmentierung zu verzeichnen. Zuerst glaubte ich natürlich, eine urämische Darmaffektion vor mir zu haben, obzwar die Lokalisation eher für leichte Ruhr bzw. Ruhrrezidiv sprach. Bakteriologisch ließen sich zahlreiche Kolonien von Y-Ruhr züchten, so daB die erste Auffassung fallen mußte.

Noch schwerer wäre ohne bakteriologische Untersuchung Fall 82 aufzuklären gewesen. Es handelte sich um einen Kranken, der nie im Felde gewesen war; seit geraumer Zeit wurde er in einem Hinterlandspital an Nephritis behandelt und erkrankte dann im März 1918 an Bauchtyphus. Nähere Aufzeichnungen über den Verlauf dieser Erkrankung stehen mir nicht zu Gebote. Am 25. IV., also beinahe 2 Wochen vor dem Tode, wurde er mit der - offenbar fingierten - Diagnose Darmtuberkulose nach Bauchtyphus in. das hiesige Spital transferiert, wo er auf eine Tuberkulosebaracke gelegt wurde. Im weiteren Verlauf bestand Fieber; Stuhlgang zwei- bis dreimal täglich. Bei der Obduktion fand ich eine nicht sehr schwere, rekrudeszierende Endokarditis und ältere herdförmige interstitielle Nephritis mit leichter narbiger Nierenschrumpfung; im Ileum waren mehrere gereinigte, in Abheilung begriffene typhöse Geschwüre, die mesenterialen Lymphknoten und Milz waren aber beträchtlich geschwollen, und aus der letzteren wíe auch aus der Galle ließen sich mit Leichtigkeit Typhusbazillen züchten. Es handelte sich offenbar um einen rezidivierenden Typhus; die Herz- und Nierenerkrankung mußte älteren Datums sein und war mit der erstgenannten Krankheit in keinem Zusammenhang. Im Dickdarm aber bestand eine diffuse Rötung und leichte Schwellung der Schleimhaut; letztere war von zahlreichen punktförmigen bis pfefferkorngroßen Blutaustritten durchsetzt, stellenweise leicht getrübt, und auf ihrer Oberfläche zeigte sich eine serös-schleimige $\mathrm{Ab}$ - 
sonderung. Auf den Endoplatten, die mit dem von der Darmwand abgekratzten Schleim beimpft wurden, fanden sich außer Colibazillen einige zarte, farblose Kolonien, die sich gegen meine Erwartung als Ruhrbazillen erwiesen ( $Y$ ). Ich kann diesen Befund nicht anders auffassen als eine im allerersten Stadium sich befindende Ruhrinfektion, die im Spital selbst stattgefunden haben mußte. Auffallend waren die zahlreichen kleinen Blutungen in der Dickdarmschleimhaut; sie sind wahrscheinlich auf die bekannte Neigung zur hämorrhagischen Diathese bei Typhus zurückzuführen.

Fall 29 betraf einen 50jährigen Mann mit Atheromatose der Aorta; äußere Genitalien und Beine waren geschwollen, außerdem bestand deutliche Atrophie sämtlicher Organe. Die Dickdarmschleimhaut war mäßig gerötet und geschwollen, im unteren Abschnitt des Colon von festhaftenden, schmutziggrauen, dicken Auflagerungen bedeckt, die sich auf die vorspringenden Falten und Buckel beschränkten und nirgends an größeren Flächen konfluierten. Die Muskulatur des Dickdarms war dünn, tiefere Substanzverluste der Schleimhaut waren nirgends zu finden. Trotzdem hatte sich diffuse Peritonitis mit dünnem serofibrinösen Exsudat entwickelt, die wahrscheinlich durch Infektion einer vorher bestehenden Aszites entstand. Anamnese und Krankengeschichte waren nicht aufgezeichnet worden; der Mann war nur 8 Tage im hiesigen Spital.

Auch hier handelte es sich um eine verhältnismäBig leichte Darmerkrankung, die aber ein schon vorher herabgekommenes Individuum befallen hatte; die Bauchfellentzündung, welche durch Infektion auf lymphatischem Wege entstanden sein mußte, hat den tödlichen Ausgang beschleunigt.

Die übrigen akuten Fälle boten keine Besonderheiten in bezug auf den Darmbefund. Beim Fall 92 lag diffuse Diphtherie des ganzen Dickdarms und des unteren Teiles des Ileum vor, verursacht durch ShigaKrusebazillen, die in zahlreichen Kolonien aufgingen, obzwar die Obduktion erst etwa 60 Stunden p. m. vorgenommen werden konnte; die Krankheit dauerte 18 Tage.

Zwei weitere Fälle von Darmdiphtherie betrafen Patienten mit schweren vereiterten Schußverletzungen; beide waren amputiert. Der eine (44) war 4 Wochen, der andere (77) bereits 6 Monate in Spitalbehandlung. Bei dem ersten sind 12 Tage vor dem Tode Durchfälle im Vormerkblatt verzeichnet, die klinischerseits als septisch aufgefaßt wurden, bei dem anderen fand ich keine Angaben über etwaige Ruhrsymptome. Die Darmaffektion hatte man vielleicht als septische Enteritis betrachten können, obzwar sonst keine Zeichen akuter Sepsis vorhanden waren. Die Darmveränderungen waren bei dem ersten wahrscheinlich etwas älter, da im Sigma schon einige sinuöse (follikuläre), anscheinend gereinigte Geschwüre bestanden, der andere mußte dagegen als Spitalsinfektion gedeutet werden.

Für die Chirurgen mögen aber diese beiden Fälle als Mahnung gelten, daß hinter den Durchfällen, die in Begleitung septischer Erkrankungen 
auftreten, einmal auch echte Ruhr stecken kann; daran ist vor allem zu denken, wenn der rapide Verfall des Patienten mit dem guten Aussehen der Wunde nicht in Finklang $\mathrm{zu}$ bringen ist.

Der überaus größte Teil meiner Fälle gehört den späteren Stadien der Ruhr an. Diese sind in erster Linie durch das Vorkommen von Geschwüren charakterisiert. Trat nämlich im Stadium der Diphtherie durch schwere Intoxikation der Tod nicht ein, so Iösen sich allmählich die Schorfe ab, mit ihnen geht die nekrotische, mehr oder weniger dicke Schicht der Sehleimhaut in Verlust, und es erscheint eine rötliche, feuchte Geschwürfläche, die oft durch einen schmalen tiefroten Saum begrenzt ist (flächenhaft fressende, diphtherische Geschwïre). Bald fängt die Heilung an. Die Ränder blassen ab und werden unscharf, verschwommen, der Geschwürgrund glättet sich, die serös-blutige Exsudation verschwindet und auch die übrige Schleimhaut schwillt ab; nach und nach bekommt das ganze Geschwür den matten Glanz der normalen Schleimhaut, von der er zuletzt nur durch die etwa zurïckbleibende Pigmentierung zu unterscheiden ist. Der Heilungsvorgang schreitet meist von oben nach unten vor, und man sieht nicht selten im Rectum und Sigma, wo die diphtherischen Veränderungen in der Regel am schwersten sind, noch ausgedehnte, zuweilen noch belegte Geschwüre, wo sehon im oberen Dickdarmabschnitt niehts mehr als Pigmentierung in der anscheinend normalen Sshleimhaut zu sehen ist. In einigen Fällen sah ich aber einen umgekehrten Vorgang, indem im unteren Dickdarm nur mehr gereinigte Geschwüre zu sehen waren, an der oberen Grenze der Affektion aber noch anscheinend frische Pseudomembranen sich fanden; ich kann aber nicht mit Sicherheit sagen, ob es sich dabei nicht um Rezidive handelte. Außer im Rectum scheint auch der Prozeß im Ileum ziemlich langsam abzuklingen (Fall 47).

Es ist klar, daß nur die relativ leichten Erkrankungen in Heilung übergehen können; die schweren Fälle, die mit tiefgreifender primärer Mortifikation der Schleimhaut einhergehen, enden meist nach kurzem Verlauf tödlich. So ist zu erklären, daß man in den protrahierten Fällen größtenteils nur oberflächliche, nicht einmal die ganze Dicke der Schleimhaut durchsetzende Geschwüre findet (Löhlein). Doch findet man zuweilen in den am schwersten affizierten Darmabschnitten, d. h. im Rectum und oft im Sigma, auch tiefere, die Musc. mucosae entblößende Substanzyerluste, die dann natürlich einer längeren Zeit zur Heilung bedürfen; hier sieht man oft, daß das Zentrum des Geschwürs noch einen Schorf trägt, wo die Ränder sich schon abzuglätten beginnen. Beitzke, der die mikroskopischen Heilungsvorgänge bei der Ruhr in schönen Untersuchungen 
aufgeklärt hat, erklärt es als eine vom Rande her fortschreitende Epithelisierung, die oft schon tief unter den Schorf reichen kann.

Die in solch glatter Abheilung sich befindlichen Fälle kommen natürlich selten zur Obduktion, sei es, daß eine Komplikation, z. B. tuberkulöse Pleuritis und ähnliches (an deren Entstehung die Ruhr selbstverständlich nicht ohne Schuld ist), den Tod herbeibringt (Fall 10, 12, 35, 73). Die bakteriologische Untersuchung des Präparates fällt dann aber meist negativ aus, ein Zichen, daß die Ruhrbazillen zu dieser Zeit bereits verschwunden sein mïssen. Das stimmt auch mit der klinischen Beobachtung überein, daß die normal verlaufenden Ruhrfälle in der Rekonvaleszenz meist negative Ergebnisse bei der Stuhluntersuchung ergeben.

Weitaus öfter sieht man bsi den zur Obduktion gekommenen Fällen den krankhaften DarmprozeB an einzelnen Stellen sich länger unterhalten. Dazu sind vor allem die tiefen, sinuösen Geschwüre geeignet, die für die späteren Stadien der Ruhr überaus charakteristisch sind, wenn sie anch manchmal fehlen können. Thre Entstehungsart ist verschieden. Wenn auch die Nekrose bei den protrahierten Fällen im allgemeinen oberflächlich zu sein pflegt, so kann sie doch an einzelnen Stellen, und das ist meist das Sigma oder Rectum, tiefer greifen und die ganze Schleimhaut mitsamt der Muscularis mucosae zerstören, oder aber es können derartige tiefe Verschorfungen durch nachträgliche eiterige Einschmelzung vertieft und erweitert werden. Ich habe sehr oft derartige ausgedehnte tiefe Geschwüre mit unterminierten Rändern in protrahierten Fällen gesehen, die durchaus die bekannte "stufenleiterähnliche" Ausbreitung aufwiesen, welche den bei Darmdiphtherie am schwersten betroffenen Quer- und Längsfalten der Schleimhaut entspricht.

Zweitens beobachtete ich oft schon bei diffuser Darmdiphtherie vereinzelte, meist im Rectum sitzende, kleine, runde, dabei aber sehr tiefe Geschwüre; in weiter vorgeschrittenen Fällen waren sie schon reiehlicher; zaigten deutlich unterminierte Rändèr und konfluierten zum Teil zu größeren Geschwüren. Zuweilen ließen sich alle Ubergänge von diesen kleinen Defekten bis zu ausgedehnten Geschwüren mit stark unterminierten Rändern feststellen. Da ich bis jetzt keine mikroskopischen Untersuchungen des aufbewahrten Materials vornehmen konnte, kann ich nicht mit Sicherheit sagen,-ob sie etwa aus vereiterten Lymphknötchen entstanden sind, ob es sich also um ,follikuläre" Geschwüre im Sinne Rokitanskys, Heubners und Orths handelt oder nicht. Löhlein bestreitet das Vorkommen von Lymphknötcheneiterung bei der Rulir und spricht ihr besonders jede Bedeutung für die Entstehung der sinuösen 
Geschwïre ab; nach seiner Ansicht handelt es sich bei den alten Beschreibungen um eine Verwechslung mit der (außerdem noch falsch histogenetisch aufgefaßten) Amöbenruhr. Neuerdings beschrieb aber Westenhöfer Fälle von unzweifelhafter primärer nodulärer Ruhr, und ich kann seine Boobachtung auf Grund einiger meiner Fälle nur bestätigen. Nun lehnt aber Löhlein die Beziehungen zwischen;den Lymphknötchen und den sinuösen Geschwüren prinzipiell nicht ab, schreibt ihnen aber nur eine indirekte Rolle zu: sie bilden die Pforte für eine Wucherung der Fundusteile von Lieberkühnsschen Krypten, die bei gewissermaßen tiefer Diphtherie durch die vorgebildeten Lücken der Muscularis mucosae in 'die Submucosae hineinwachsen, wo sie dann durch Schleimretention die bekannten Sshleimzysten bilden. Die sinuösen Geschwüre und submukösen Abszesse sollen aber durch Vereiterung dieser dystopischen Krypten entstehen. Ich kann zu dieser Streitfrage keinen Standpunkt einnehmen, da ich vorläufig über mikroskopische Untersuchungen nicht verfüge; was ich aber hervorheben möshte und was mir für das makroskopische Aussehen der Geschwüre wesentlich $z u \cdot$ sein scheint, ist die Tatsache, dab, wenn einmal die auf die eine oder andere Weise entstandenen' Gesehwüre die natürliche Barriere der Schleimhautmuskelschicht durchbrechen, dann immer eine Tendenz zu mehr oder weniger rascher Ausbreitung des Prozesses im submukösen Gewebe zutage tritt, was natïrlich durch die lockere Beschaffenheit der Submukosa, die dem Fortschreiten der Eiterung wenig Widerstand leistet, leicht zu erklären ist. So kommt es zu den bekannten submukösen Eiterungen, die dann unter Bildung langer Fistelgänge die Schleimhaut sekundär durchbrechen können oder sich mit benachbarten!Gesehwüren urterhalb der Sehleimhaut vereinigen und diese auf große Strecken unterminieren, so daß sie von der Unterlage abgelöste Brücken, Spannen und Fetzen bildet, die dann infolge mangelhafter Ernährung der Nekrose verfallen. Auch die Muskulatur der Darmwand wird zuweilen arrodiert, und es kann, wenn auch selten, zu Perforation kommen. Ob diese Eiterung noch als Wirkung der Ruhrbazillen anzuschen ist, erscheint mir zweifelhait; ich glaube im Einklang mit Löhlein, daß sie eher auf sekundäre Infektion zurückzuführen ist, da ich von den Abszassen meist Kokken, aber nur selten Ruhrbazillen ziichten konnte.

Nicht immer kommt es zu derartig ausgedehnten Verwüstungen; doch sind die Ränder der in der Submukosa reichenden Geschwüre nach Eliminieren des nekrotischen Gewebes stets mehr oder weniger stark unterminiert.

Wenn sich diese Geschwüre einmal ausgebildet hatten, so ist es 
kaum möglich, zu einigermaßen sicherem Aufschluß über ihre Entstehungsweise zu gelangen. So kommt man bei dem Versuch um eine Klassifikation der im Spätstadium der Ruhr vorkommenden Geschwüre nicht über die rein morphologische Unterscheidung zwischen den flächenhaften, oberhalb der Musc. mucosae liegenden und den tiefen sinuösen Geschwüren hinweg. Die Heilung dieser Geschwüre geht natürlich nicht in so einfacher Weise vor sich, wie jene der oberflächlichen. Manchmal kleben zwar die Ränder wieder auf die Unterlage an und es kommt zu allmählicher Uberhäutung der Geschwürfläche duirch neugebildete Schleimhaut, deren epitheliale Komponente von der angrenzenden gesunden Schleimhaut geliefert wird (Beitzke), das andere Mal wird aber die untere Fläche der überhängenden Ränder von der Sehleimhaut überhäutet und so ein glattes Anwachsen derselben verhindert; das ist meines Erachtens hauptsächlich dann der Fall, wenn der gereinigte Geschwürgrund von der bloßgelegten, nur spärliches Granulationsgewebe produzierenden Muskelschicht gebildet wird. Haben sich die gegenüber liegenden Ränder durch krampfhafte Kontraktion des Darmes genähert, so können sie unter Umständen miteinander verkleben, und durch die Wucherung und Schleimretention der darunter abgeschlossenen Krypten kommt es später zur Bildung umfangreicher, oft tief in der Submukosa liegender Schleimzysten (Orth, Beitzke).

Durch bloß makroskopische Beobachtung kann man natürlich die Heilungsvorgänge nur annähernd beurteilen; sicher ist aber, daß die sinuösen Geschwüre recht langer Zeit zur völligen Ausheilung bedürfen, wie das aus den Befunden bei einigen meiner Fälle klar hervorgeht (z: B. Fall 13). Makroskopisch findet man die Ränder dieser tiefen Geschwüre meist geröter und geschwollen, wo die übrige Schleimhaut schon völlig abgeblaßt ist. Liegen die Geschwüre nahe beieinander oder koinfluieren sie, so können die zwischen ihnen stehen gebliebenen Schleimhautbrücken und -inseln stark geschwollen und tiefrot verfärbt scin, so daß sie als polypenartige Gebilde imponieren.

Dabei scheinen die Geschwüre manchmal nur geringe Symptome zu verursachen; ich habe nicht selten ausgedehnte Geschwüre als Nebenbefund bei Fällen entdeckt, wo der Kliniker nicht den geringsten Verdacht auf das Vorhandensein von Ruhr äußerte. Damit stimmt auch eine Beobachtung von Singer überein, der bei völlig symptomlosen Rekonvaleszenten nach Ruhr rektoskopisch ausgedehnte Ulzerationen nachweisen konnte. Ähnliche Beobachtung haben übrigens schon frühcr Lentz und Kantorowitz gemacht. Für das Chronischwerden scheinen sie aber in einer anderen Richtung von Bedeutung zu sein, worauf ich noch weiter unten zu sprechen komme. 
In den weitaus meisten Fällen beschränken sjch die Befunde bei protrahiertem Verlauf nicht auf diese beiden Arten von Geschwüren, sondern man findet mehr oder weniger ausgedehnte frische Veränderungen von verschiedener Intensität, wie Rötung, Schwellung und Blutungen, fibrinöse Exsudation in Form von kleinen Auflagerungen bis zusammenhängenden Pseudomembranen und tiefe Verschorfungen sowohl auf der erhalten gebliebenen Schleimhaut wie auch ïber den kaum abgeheilten flachen Geschwüren (61). Meist bleiben diese Erscheinungen in bezug auf Intensität und Ausdehnung hinter jenen bei akuten Formen weit zurück, zuweilen findet man aber, neben einigen Narben und Pigmentierungen oder Schleimzysten, die auf ältere Krankheitsstadien hinweisen, fast zusammenhängende Diphtherie, die von einer akuten Ruhr sehwer zu unterscheiden ist $(4,90)$. Das andere Mal sind neben ausgedehnten Vernarbungen im unteren Dickdarmabschnitt nur ganz geringe katarrhalische Erscheinungen auf einer Stelle, z. B. oft im Coecum, zu verzeichnen (83). Diese akuten Veränderungen können schon wieder im Abklingen vorgefunden werden, oder sie heilen auf dem einen Ende des betroffenen Darmabschnittes ab, um auf dem anderen Ende weiter fortzusehreiten; in seltenen Fällen findet man die älteste Partie, wo die Geschwüre bereits in Abheilung begriffen sind, von einer zweiten Wolle des Rezidivs betroffen.

Diese Wellen schreiten meist in aufsteigender Richtung fort; am schwersten pllegt auch hier das Rectum ergriffen zu sein, wo in extremen Fällen nach Verlust der ganzen Schleimhaut die Innenfläche des Darmrohres eine einzige narbige Fläche darstellt, die aber, soweit es sich makroskopisch beurteilen lüßt, doch wieder vom Epithel ansgekleidet werden kann; dann ist sie glatt oder höckerig, schiefergrau, derb, ohne Falten $(76,83)$. Die stehen gebliebene Schleimhaut (soweit sich das feststellen läßt) weist oft produktiv entzündliche Veränderungen auf; sie ist verdickt, unregelmäßig granuliert oder auch schon geschrumpft, lederartig.

Sshließlich gehören noch zu dem Bilde der chronischen Ruhr die bereits oben erwähnten Schleimzysten, die oft in großer Zahl in der Submukosa sitzen, die Schleimhaut in Form von kleinen Buckeln hervorwölben und durch die aus ihnen hervorragenden, froschlaichähnlichen Schleimklumpen dem Ganzen ein höchst merkwürdiges Aussehen verleihen: Ich habe oft sehr markante Bilder solcher Colitis cystica profunda gesehen, wo keine tiefen Geschwüre vorhanden waren; die Muscularis mucosae war hier anscheinend erhalten geblieben $(18,19,50,79)$. Entweder waren hier die Geschwüre bereits verheilt und die Zysten stellen ihre einzigen Reste vor oder aber entstanden die letzteren durch primäre Tieferwucherung der Krypten in die Submukosa hinein. "Die kürzeste Krank- 
heitsdauer, nach welcher ich solche Zysten sah, war etwa zwei Monate (Fall 28).

Durch das Neben- und Ubereinander dieser Veränderungen, wobei natiirlich im gegebenen Falle einzelne Komponenten fehlen können, kommt das äußerst mannigfaltige und komplizierte Bild der chronischen Ruhr zustande, das oft nicht leicht zu interpretieren ist. Insbesondere ist es fast unmöglich, etwaige Rückschlüsse auf das Alter und die Verlaufsart der Erkrankung zu ziehen. Es wäre ein sehr dankbares Unternehmen gewesen, die anatomischen Veränderungen mit dem klinischen Verlauf zu vergleichen, wären die mir zur Verfügung stehenden klinischen Aufzeichnungen nicht so sehr dürftig und lückenhaft gewesen, daß man sich aus ihnen nur selten eine etwas klare Vorstellung von dem Krankheitsverlauf machen konnte. Es ist aber von vornherein klar, daß von dem Vorhandensein frischer katarrhalischer oder diphtherischer Prozesse (Rezidiv) das Bastehenbleiben oder Wiederauftreten schleimig-blutiger Durchfälle abhängig sein muß.

Die Krankheitsdauer meiner chronischen Fälle, soweit sie zu ermitteln war, bewegte sich zwischen einigen Wochen und mehreren Monaten. In einem Falle (40) betrug sie 7 Monate, ohne daß die vorgefundenen Veränderungen ein so langes Bestehen des Prozesses ahnen ließen; sie glichen vielmehr dem späteren Stadium einer frischen Erkrankung. In zwei anderen Fällen von 7 monatiger Dauer fand ich dagegen ausgedehnte narbige Verwüstungen der Sehleimhaut $(76,83)$. [In einem noch älteren Fall (78) handelte es sich um eine Kombination mit Tuberkulose.] Auch in den übrigen Fällen ließen sich keine gesetzmäßigen Beziehungen zwischen dem Aussehen des Präparates einerseits und der Krankheitsdauer andererseits aufstellen; zuweilen fiel ein solches Mißverhältnis zwischen den vorgeschrittenen, anscheinend sehr alten Veränderungen und der kurzen Krankheitsdauer auf, daß man notwendig auf das Vorhandensein einer viel älteren, aber latent verlaufenden und erst durch Eintritt eines Rezidivs manifest gewordenen Ruhr denken mußte, was um so wahrscheinlicher erscheinen mußte, als sich die Anamnesen auf früher durchgemachte Krankheiten zumeist nicht bezogen.

Da auch die weitschweifigste Schilderung das individuelle Gepräge des Einzelfalles nicht wiedergeben kann, nehme ich von der Beschreibung der einzelnen Befunde Abstand. Auf einige bemerkenswerte Einzelheiten und auf die mit anderen Krankheiten kombinierten Ruhrfälle komme ich noch zu sprechen.

Daß ich aber die Pathogenese der chronischen Ruhr im allgemeinen etwas ausführlicher schilderte, obzwar ich mir bewußt bin, nicht viel 
neues sagen zu können, geschah hauptsächlich aus dem Grunde, daß diese anatomischen Tatsachen zu der Auffassung der chronischen Ruhr von Jürgens im schroffen Gegensatz stèhen.

Der genannte Autor gibt zwar zu, daß nach Ablauf der akuten Ruhr manchmal Rückfülle vorkommen können, schließt diese aber von dem Begriff der eigentlichen chronischen Ruhr aus.

Letztere ist nach Jürgens „eine Ruhr, die nicht in wenigen Wochen abläuft und heilt, sondern längere Zeit, etwa monatelang, Beschwerden und. Gesundheitsstörungen hinterläßt. Ganz selbstverständlich ist es auch, daB die Erscheinungen der chronisehen Rubr anders sein müssen als die der akuten Krankheit, denn die Allgemeinerscheinungen der Ruhr lạufen wie die jeder akuten Infektionskrankheit in kurzer Zeit $a b$, und auch das augenfälligste Symptom der Ruhr, der schleimig-blutige Ruhrstuhl, ist die Folge einer der Ruhr eigentümlichen Darmveränderung, die sich rasch entwickelt, um sich ebenfalls rasch wieder zurückzubilden, und die jedenfalls in ihrer diphtherischen Form nicht in ein chronisches Stadium übergehen kann."

„Von der chronischen Ruhr kann nur dann gesprochen werden, wenn nach" Ablauf der eigentlichen Infektionskrankheit Störungen zurüekbleiben, die eine völlige Genesung auf lange Zeit verzögern und nicht selten die Ursache schweren Siechtums werden können. In solchen Fällen fehlen die eigentlichen Ruhrsymptome vollständig. Die Ruhr ist abgelaufen, Schädigungen des Darmes verzögern oder verhindern aber die völlige Genesung." Die blutig-schleimigen Durchfälle verschwinden, im weiteren Verlauf finden sich breiige oder flüssige Stühle mit Zeichen der Dünndarmdysenterie.

„Das sind also Zeichen, die nichts mehr mit der eigentlichen Ruhr zu tun haben und wahrscheinlich auch gar nicht als Ausdruck des vielleicht noch bestehenden bazillären Infektes, sondern als Folge der abgelaufenen Darmerkrankung zu deuten sind.“

Weiter unten definiert er chronische Ruhr, ,als ein lokales Leiden mit zeitweise schleimig-blutigen Durchfällen, dem jede Kennzeichnung als akute Infektionskrankheit und auch jede Neigung zu epidemischer Ausbreitung fehlt".

Dem zuwider muß ich hervorheben, daß bei der chronischen Ruhr in den weitaus meisten Fällen Veränderungen vorhanden sind, die sich von den im akuten Stadium vorkommenden höehstens durch ihre geringere Intensität und Ausdehnung unterscheiden, die also schon anatomisch als Rückfälle bezeichnet werden müssen. Dieselbe Auffassung teilt auch Löhlein; er betont auch die spezifische Natur dieser Rezidive, ohne aber dafür Beweise zu geben: Meine Untersuchungen zeigten, daß man dort, wo solche frischen Prozesse bestehen, aus den befallenen Partien der.Schleimhaut, und of nur aus diesen, Ruhrbazillen mit soleher Regelmäßigkeit züchten kann, daß 
an eine Zufälligkeit der Befunde nicht zu denken ist; sic beweisen, dab diese Prozesse spezifischer Natur, $d . h$. durch die Wirkung des Ruhrbazillengiftes bedingt sind und deshalb als echte Rückfälle aufgefaßt werden müssen. Natürlich kann ein solcher Prozeß nach wiederholtem Aufflackern schließlich doch zum Stillstand kommen. Man findet zuweilen bei stark kachektischen Leichen im Dickdarm nur in mehr oder weniger vorgeschrittener Abheilung begriffene Geschwüre, pigmentierte Narben oder Schleimzysten, aber keine Zeichen eines frischen Prozesses, ohne daß dic vorhandene Kachexie durch ein anderes Leiden erklärt werden könnte; hier pflegt auch der bakteriologische Befund negativ zu sein (z. B. Fall 18, 76, 83). Danit soll aber nicht gesagt werden, daß es sich hier um Uberreste einer einzigen akuten Attacke handeln müßte; man muß im Gegenteil annehmen, daß die ausgedehnten Verwüstungen und Vernarbungen das Rosultat wiederholter Rezidive vorstellen. Auch die lange 'Krankheitsdauer (in Fall 186 Monate, in Fall 76 und 837 Monate) spricht eher für diese Auffassung. Experimentelle Erfahrung lehrt, daß mit Ruhrbazillen oder ihren Toxinen wiederholt behandelte Tiere oft unter Zeichen fortschreitender Kachexie zugrunde gehen; wenn man auch die Resultate des Tierversuches ohne weiteres auf den Menschen nicht übertragen darf, so scheint es doch wahrscheinlich, daß es sich bei der Ruhrkachexie mit geringen Lokalsymptomen des Darmleidens um ähnliche Wirkungsweise der Ruhrbazillengifte handeln kann. Viel ölter findet man derartige abgelaufene Ruhrprozesse als Nebenbefunde bei 'Kranken, die an einer interkurrenten Krankheit starben (63) oder einem durch die schwere Schädigung begünstigten chronischen Leiden, wie Tuberkulose, erlegen sind (Fall 21, 27, $72,73,86)$.

In einem Punkte kann ich Jürgens beistimmen, daß nämlich die tiefen geschwïrigen Prozesse in den engeren Rahmen der Ruhrbazillenwirkung nicht gehören, daß sic vielmehr nur als Uberreste derselben aufzufassen sind, wobei aber sekundäre Infektionen meist mit im Spiele sind.

Weiter unten liest man: „,Manchmal entstehen tiefer greifende Prozesse, deran Heilung nicht so rasch und nicht so vollständig vor sich geht. Solche Veränderungen sind es nun, die manchmal unter dem Einflu $B$ anderer Schädlichkeiten zu neuen "Krankheitserscheinungen führen können." Im hiesigen Spital kam es sehr oft vor, daß sich Leute, die sich daheim auf Urlaub befanden, mit schleimig-blutigem Durchfall krank meldeten. Seitens der Militärärzte wurde der Verdacht ausgesprochen, daß es sich um künstlich erzeugte Erkrankungen handelte. Und doch zeigte der weitere Verlauf und der oft positive Stuhlbefund, daß echte 
Ruhr im Spiele war. Ich glaube eine bessere Erklärung dieser Erschejnungen geben zu können: Es handelte sich eben um Leute mit chronischer latenter Ruhr, die aber, durch lange Erfahrung belehrt, sehr wohl wissen, daß und welche äußere Sshädlichkeiten, sei es ein Diätfehler oder vielleicht ein drastisches Abführmittel, bei ihnen Wiederauftritt der Ruhrerscheinungen hervorruft, und die nicht anstehen, sich dessen zu bedienen, wenn es sich darum handelt, der Wiedereinrückung ins Feld auszuweichen.

Im weiteren führt Jürgens aus: „Allerdings sind wir über die Beziehungen der Rubrbazillen zum Körper noch sehr ungenügend unterrichtet, und es ist für uns nicht gleichgültig, welche Rolle die Bazillen nach Ablauf der Ruhr im Darm spielen, aber diese Fragen werden nicht dadurch gefördert, da $\dot{B}$ geschwürige und nekrotisierende Prozesse ohne weiteres auf Rechnung der Ruhrbazillen gesetzt werden."

Nun glaube ich bewiesen zu haben, daß wenigstens ute nекrousierenden Prozesse auf Rechnung der Ruhrbazillen gesetzt werden müssen; es drängt sich aber die Frage auf, wo sich die letzteren während der Latenzzeit im Darme verstecken. Ein Zufall hat mir es ermöglicht, diese Frage gewissermaßen aufzuklären. Der Fall (13) sei hier etwas ausfiuhrlicher besprochen.

Sch. M., 46 J., hat im März 1916 Rheumatismus durchgemacht. Am 29. V. 17 erkrankte er unter Ruhrsymptomen; es bestand Fieber und blutig-schleimiger Durchfall. Zugleich zeigten sich aber Symptome einer Endokartitis und später stellten sich Odeme ein. Die Darmsymptome sind später allmählich zurückgegangen, um schließlich völlig zu verschwinden, und der Patient fühlte sich relativ wohl; am 9. VII. trat plötzlicher Tod ein.

Bei der Autopsie fand ich eine akute Nephrose, die wahrscheinlich die Todesursache war, außerdem eine verruköse Endokarditis. Querkolon war stark gebläht, etwas schwächer der absteigende Dickdarm; Sigma und Rectum schmal. Im letzteren befanden sich in der Schleimhaut einige runde, ganz kleine Grübchen mit flachen oder etwas vorgewölbten, leicht grau pigmentierten Rändern; am senkrechten Schnitt sah man, $\mathrm{daB}$ sie bis in die Submukosa reichen, wo sie sich buchtenartig erweitern. Die meisten dieser Rezesse enthielten etwas Ejter; in ihrer Umgebung waren keine Zeichen akut entzündlicher Reaktion festzustellen; auch die Schleimhaut war völlig blaß. Im Sigma dieselbe Veränderung; weiter oben waren die Öffnungen immer weiter, so daß sie schließlich als tiefe sinuöse Geschwüre erschienen. Im Querkolon waren dann diese Defekte recht zahlreich und auch viel größer;' stellenwejse sah man deutlicke Zeichen einer Zerrung, welche wohl durch die starke Blähung des Dickdarmes zu erklären war. Der Grund der Geschwüre, die meist bis auf die Muscularis reichten, war vollständig gereinigt, die Ränder etwas unterminiert, aber nicht geschwollen, glatt; auch die übrige Schleimhaut wax durchaus bla $B$, von normalem Aussehen. 
Es handelt sich offenbar um eine glatt obgeheilte akute Ruhr, deren Reste bei dem an einer akuten Nephrose verstorbenen Patienten als zufälliger Nebenbefund zu deuten sind. Die Nephrose war aber wahrscheinlich durch die Endokarditis verursacht, die ihrerseits wieder mit dem anamnestisch ermittelten Rheumatismus im ursächlichen Zusammenhang war. Die Entstehungsart der Darmgeschwüre läßt sich natürlich bei dem vorgeschrittenen Entwicklungsstadium derselben schwer erkennen; einige jener kleinen, mit Eiter erfüllten Rezesse im Rectum habe ich in Schnitten untersucht, darin aber keine epithelialen Bestandteile gefunden. Wahrscheinlich handelte es sich um follikuläre Geschwüre im Sinne Rokitawkys, die aber sehon. wieder in Abheilung begriffen waren.

Aus dem Eiter mehrerer der erwähnten Rezesse im Rectum lieben sich aber Ruhrbazillen. fast in Reinkultur züchten.

In drei anderen Fällen von Colitis cystica konnte ich Ruhrbazillen aus Schleimzysten züchten $\left(50^{1}, 79,80\right)$; hier lagen aber die Verhältnisse nicht so klar, da die Veränderungen einen rezidivierenden Charakter hatten.

Diese Befunde flößen den Gedanken ein, daß die in den Rezessen und Zysten versteckten Bazillen durch verschiedene auf den Zustand des Darmes einwirkende Momente mobilisiert werden können und so eine Wiedererkrankung der Schleimhaut hervorrufen; wie es aber kommt, daB sie innerhalb der Zyste ihre toxische. Wirkung nicht entfalten, bleibt zu erklären. Man darf sich allerdings die Sache nicht so grobmechanisch vorstellen, denn sicher spielen bei dem Zustandekommen der Rezidive auch immunisatorische Prozesse eine Rolle; dafür spricht einerseits der Verlauf der akuten, bald abheilenden Erkrankung, andererseits die geringere Intensität der rezidivierenden Prozesse im Vergleich mit den ersteren. Ich stelle mir die Sache so vor, daß die akute Erkrankung einen gewissen Grad von Immunität hinterläßt, die in der Rekonvaleszenz allmählich wieder abklingt, bis sie schließlich so schwach wird, daß die Ruhrbazillen, welche inzwischen in einer Schleimzyste, vor der Konkurrenz der Darmsaprophyten relativ geschützt, sich erhalten hatten (wo zudem für die Resorption ihrer Gifte die Verhältnisse nicht günstig liegen), ihre pathogene Wirkung wieder entfalten können.

Damit soll natürlich nicht gesagt werden, daß die Sache in allen Fällen unbedingt in dieser Weise vor sich gehen muß. Es gibt Fälle, wo nach Ablauf des akuten Stadiums die Ruhrerscheinungen eigentlich nie

1 Hier allerdings ein atypischer Stamm. 
zum Stillstand kommen. Hier liegt vielleicht die Sache so, daß die erreichte Immunität zwar genügt, um die Wirkung der Ruhrgifte auf den Gesamtorganismus za verhindern, doch aber nicht stark genug ist, um die lokal toxische Komponente der Bazillen unschädlich zu machen; so unterhält sich der Ruhrprozeß im Darm mit abwechselnder Intensität so lange, bis schließlich doch Heilung eintritt oder aber die fortdauernde Uberschwemmung des Organismus mit Toxinen' gemeinsam mit der Schädigung der Darmfunktion zur sehweren Kachexie und schließlich zum Tode führt.

Ioh bin mir wóhl bewußt, daß diese Ansicht nichts weiter als eine Hypothese ist, die erst zu beweisen wäre; demjenigen aber, der diese Frage auf experimentellem Wege in Angriff nehmen wollte, könnte sic sehr gut als Arbeitshypothese dienen.

Was aber aus meinen Untersuchungen klar hervorgeht, das ist die Infektiosität des chronisch Ruhrkranken, der, wenn er auch lange Zeit völlig symptomlos war, schließlich doch wieder an einer anscheinend harmlosen Diarrhoe erkranken und seine Umgebung infizieren kann. Ich weiß, daß ich damit nichts Neues sage, denn durch chronisch Ruhrkranke verursachte Epidemien wurden schon vielfach beschrieben (Negri und Panc, Pfuhl, Eckert, Lentz, Kruse, Luksch, Küster, Hawkins, Mayer; Boehnke u. a.). Lentz spricht sich über diesen Punkt folgendermaßen aus:

„Epidemiologisch noch wichtiger als diese oben erwähnten gesunden Bazillenträger sind die Ruhrbazillendauerausscheider. Mit dem Eintritt der klinischen Genesung hört nämlich bei der Ruhr ebensowenig wie beim Typhus die Ausscheidung der Krankheitserreger immer auf. Vielmehr erstreckt sich diese häufig noch wochenlang in die Rekonvaleszenz hinein, und ein gewisser Prozentsatz der erkrankt Gewesenen scheidet noch monate- und jahrelang Ruhrbazillen aus. Allerdings scheint es sich nach den bis jetzt vorliegenden Nachrichten bei dieser Dauerausscheidung nicht um eine regelmäßige Ausscheidung bei vollkommenem Wohlbefinden zu handeln, sondern häufiger um eine in mehr oder weniger unregelmäßBigen Intervallen bei Gelegenheit leichter Rezidive auftretende Ausscheidung bei chronisch ruhrkranken Individuen. Gerade diese leichten Rückfälle werden aber deshalb besonders gefährlich, weil sie meist nicht beachtet, noch viel weniger aber als Ruhrfälle gewürdigt werden. So kommit es denn, daß jeder dieser Dauerausscheider unter günstigen Bedingungen ein neues Ansteckungszentrum bilden kann."

Bis jetzt war aber diese Anschauung meist nur auf Stuhluntersuchungen gestützt, die bekanntlich bei chronischen Fällen selten zum Ziele führen, wenn man auch hier durch Anwendung einer besonders sorgfältigen 'Technik die Resultate bedeutend verbessern kann (Schürer und Wolff, Ungermann und Jötten). Ich glaube sie durch meine Unter- 
suchungen auf festere Basis gestellt $z u$ haben, vor allem durch den Beweis, daß die Chronizität des Leidens hauptsächlich durch den rezidivierenden Charakter desselben bèdingt ist und daß diese Rezidive spezifischer Natur sind.

Die praktische Bedeutung dieser Tatsache ist nicht gering. Wenn auch das Vorkommen von gesunden Bazillenträgern nicht von vornherein geleugnet werden kann ${ }^{1}$, so glaube ich doch, in Utbereinstimmung mit Dressel und Marchand, Schürer und Wolff, Kathe u. a., daß bei der Entstehung von Epidemien den chronisch Ruhrkranken eine viel größere Rolle beizumessen ist. Die große Zahl der Fälle, bei welchen erst bei der. Obduktion chronische Ruhrveränderungen als Nebenbefund aufgedeckt werden, läßt aber vermuten, daß man in der. nächsten Zeit mit derartigen herdförmigen Ruhrepidemien noch viel zu tun haben wird, die durch solche mit unbemerkt gebliebener chronischer Ruhr behafteten Personen verursacht werden.

Unter meinen Fällen von ehronischer Ruhr befinden sich nur 16, die (abgesehen von den offensichtlich sub finem entstandenen Bronchopneumonien) mit einem anderen schweren, selbständigen Leiden nicht kombiniert waren. Sämtlichen diesen Fällen war eine mehr oder weniger ausgesprouhene, manchmal exzessive Atrophie gemeinsam. Ein großer Teil derselben war mit Ödemen behaftet; manchmal bestand allgemeine Wassersucht.

Nach Jürgens, Galambos und Prym sollen bei idiopathischen, durch Unterernährung bedingten Ödemkrankheiten diphtherische oder geschwürige Darmveränderungen vorkommen, die mit Dysenterie nichts zu tun haben. Ich konnte bei einem Teil der Fälle mit Odem Ruhrbazillen nachweisen $(28,30,93,96)$; was die übrigen betrifft, wo keine Ruhrbazillen gefunden werden konnten $(6,8,24)$, zwingt mich sowohl der anatomische Befund, der für Dysenterie durchaus typisch war, wie auch der klinische Verlauf zu der Annahme, daß hier die Darmveränderungen das Primäre waren; bei einem dieser Fälle (6) war außerdem der Ruhrwidal mit Flexnerbazillen $1: 640$ positiv. $\mathrm{Ob}$ aber bei dem Zustandekommen der Hydrämie nicht auch andere Momente mitspielen, also vor: allem Unterernährung, entzieht sich meinem Urteil; auffallen mußte, daß in anderen Fällen mit nicht weniger schweren Darmveränderungen jede Andeutung von Odemen fehlte. Einige der Fälle mit ,hydrämischer Ruhr“ zeigten anatomisch ausgesprochene chronische Gastritis oder Gastroenteritis; dieselbe Veränderung fand ich aber ebenso häufig bei nichthydrämischen

1 Ford fand in 10 von 50 Leichen mit normalem Darmbefund Pseudo. ruhrbazillen, Wolf dagegen, der 24 Leichen (von Kindern) untersuchte, nur bei 3 mit deutlichem Diokdarmkatarrh. 
Fällen. Reine Odemkrankheit habe ich übrigens hierorts sehr selten auf dem Obduktionstisch gesehen.

Bei den übrigen Fällen war diè chronische Ruhr mit anderen mehr oder weniger schweren 'Krankheitsprozessen kombiniert. Es war natürlich nicht immer möglich, zu entscheiden, ob die Kachexie und der Tod auf Kosten der Ruhr oder der begleitenden Krankheit zu schreiben ist. Manchmal war sicher beides im Spiele, das andere Mal waren aber die Darmveränderungen so gering oder in so vorgeschrittenem Heilungsstadium, daß man șie bloß als Nebenbefund betrachten mußte.

In einem Falle (47) handelte es sich um rein zufällige Kombination mit einer vereiterten Schußverletzung. Hier war die chronische Ruhr (positiver Bazillenbefund) sicher die Todesursache, denn die Phlegmone war bereits im Abklingen und anatomisch waren keine Zeichen einer Sepsis zu sehen. Trotzdem wurde die Erkrankung klinischerseits als Sepsis gedeutet. Im Fall 72 hat sich in den letzten 14 Tagen Lungengangrän und jauchige Pleuritis entwickelt, die als Todesursache anzusehen war; aber auch die Darmaffektion war hier sehr schwer.

Die häufigste Kombination war jene mit Tuberkulose. Das kann nicht wundernehmen, denn die schwere Sehädigung des Organismus durch Ruhr schafft sicher einen günstigen Boden für die Tuberkulose; dabei handelte es sich zum Teil um Aktivierung eines allem Anschein nach älteren Prozesses, zum Teil aber sicher um Neuinfektion. Es waren seröse bis eiterige Pleuritiden, käsige Pneumonien und gewöhnliche ulzeröse Phthisen; einigemal Peritonitis, Miliartuberkulose und Knochentuberkulose. Die Darmveränderungen waren zum Teil recht gering, einigemal fast abgeheilt $(72,73)$, oft bestanden aber auch noch nach einem sehr langen Verlauf frische Prozesse, und der Nachweis von Ruhrbazillen gelang (Fall 407 Monate, Fall 626 Monate). Derartige Fälle sind der beste Beweis, wie lange sich floride Ruhrprozesse, ohne an sich besonders schwer sein zu müssen, im Darm erhalten können.

Handelt es sich um offene Lungenphthisen, so können sich sekundäre Darmgeschwüre nicht nur mit der gewöhnlichen Lokalisation im Ileoeoecum, sondern auch inmitten der dysenterischen Veränderungen bilden. Ieh sah öfters im Rectum und Sigma neben den gewöhnlichen flachen Ruhrgeschwüren einige tiefere, die durch ihre unregelmäßigen, wie angenagten Ränder und dureh den stark granulierenden und zugleich vernarbenden Grund auffielen (Fall 68); bei makroskopischer Beurteilung kann man derartige Geschwüre nur dann mit gewisser Sicherheit als tuberkulös ansprechen, wenn man sie auch weiter oben, außerhalb der Ruhrveränderungen, verfolgen kann. Der Gedanke liegt aber nahe, daß sich Tuberkelbazillen auf dem durch Ruhr vorbereiteten Boden besonders 
leicht niedersetzen können; so entstehen tuberkulöse Geschwüre. auch an Stellen, wo. man sie sonst selten vorzufinden pflegt. Das an sich schon verwickelte anatomische :Bild der Ruhr wird dadurch natürlich noch weiter kompliziert.

Ein seltsamer Fall (78) dieser Art sei näher besprochen. M. O., ottomanischer Soldat, litt seit 16 Monaten an blutig-schleimigem Durchfall von wechselnder Intensität; in den letzten 2 Monaten bat sich außerdem eine exsudative Pleuritis entwickelt. Wiederholte bakteriologische Stuhluntersuchung fiel negativ aus.

Der Obduktionsbefund lautete: Offene Tuberkulose der Lungenspitzen beiderseits; rechts frische serofibrinöse Pleuritis, links Reste einer abgelaufenen Pleuritis. Die Darmmuskulatur war im Rectum und in der Flexura linealis etwas verdickt; die Schleimhaut in den unteren drei Vierteln des Dickdarmes ungleichmäBig granuliert, stellenweise etwas gerötet, im allgemeinen sehr dünn; auch die Submukosa zeigte sich meist sehr dünn, offenbar narbig geschrumpft, so daß die Faltenbildung der Schleimbaut sehr eingeschränkt war.' Im Rectum, Sigma, weiter im Colon ascendens und Coecum waren einige bis hellergroße Geschwüre, die bis an die Muskularis reichten; sie besaßen deutlich unterminierte, sehr unregelmäßig konturierte, zackige Ränder; der Grund war zum Teil gereinigt, zum Teil mit wuchernden Granulationen bedeckt. Außerdem waren zahlreiche, etwa linsengroße Verschorfungen der Schlejmbaut von bröckeligem Aussehen vorbanden; dort, wo die Schorfe bereits abgestoßen waren, zeigten sich Substanzverluste, die meist bis auf die Muskularis reichten, aber wegen der Dünne der Mucosa und Submucosa seicht erschienen; ihre Ränder waren ziemlich geradlinig, ganz leicht unterminiert. Derartige Geschwüre waren massenhaft vorhanden; stellenweise konfluierten sie miteinander.

Hier war es wirklich schwer zu sagen, inwieweit die Veränderungen auf Kosten der Ruhr- oder der Tuberkelbazillen zu rechnen sind. Dagegen wies einerseits der diffuse chronisch entzündliche, zur Schrumpfung der Schleimhant und der Submukosa führende Prozeb, andererseits der positive. Nachweis von Ruhrbazillen in den Schorfen auf die dysenterische Natur der Affektion hin; die erstgenannten größeren Geschwüre waren aber höchstwahrscheinlich tuberkulöser Natur.

Daß sich Tuberkelbazillen in dem dysenterischen Darm etablieren können, zeigen zwei weitere Fälle.

Hier war die Schleimhaut des unteren Dickdarmabschnittes von zahlreichen Narben und Pigmentierungen durchsetzt, die auf eine abgelaufene Ruhr hinweisen; frische dysenterische Prozesse waren dagegen nirgends zu finden. Dafür war bei dem einen in der Flexura licualis, bei dem anderen an der Grenze des Rectum und Sigma eine mehrere Zentimeter lange Darmpartie von einem zirkulären, stark stenosierenden chronischtuberkulösen Geschwür eingenommen. Bei dem letzteren bestand außer- 
dem offene Spitzentuberkulose; das Geschwür war wahrscheinlich sekundärer Natur. Der erstere wies zwar eine Ausbreitung der Tuberkulose in verschiedenen Organen, vor allem in den Lymphdrüsen, auf, in den Lungen waren dagegen keine älteren Veränderungen zu finden; hier war die Darmtuberkulose böchstwahrscheinlich primär. Die Ruhr war bei beiden längst geheilt, die Tuberkulose aber verblieb. ${ }^{1}$

Eine interessante Kombination war ferner jene mit Malaria (16 Fälle). Die Infektion wurde ohne Ausnahme in Albanien akquiriert; es handelte sich durchwegs um Tropica.

Bei einem Teil der Fälle manifestierte sich diese Krankheit nur durch mehr oder weniger beträchtliche Schwellung und schwarzbraune Verfärbung der Milz und der Leber; im Milzausstrich war reichliches Pigment, aber keine Parasiten zu finden. Davon waren drei Fälle mit chronischer rezidivierender Ruhr kombiniert (Fall $58 \quad 31 / 2$ Monate, Fall 61 4 Monate, Fall 686 Monate). Hier war der bakteriologische Betund im Darm positiv (Fall 58 war außerdem mit einer verrukösen Endokartitis und interstitiellen Herdnephritis, Fall 68 mit käsiger Pneumonie und tuberkulösen Darmgeschwüren kombiniert). Bei einem weiteren Fall (63) war auch die Ruhr in Abheilung begriffen; eine interkurrente kruppöse Pneumonie brachte den Tod herbei. Bei einem anderen (74) mußte die Ruhr ebenfalls als abgelaufen betrachtet werden; hier war die Todesursache wahrscheinlich in der vorgefundenen schweren subchronischen Glomerulonephritis zu suchen. Ein dritter Fall (59) mit ebenfalls abheilenden Ruhrveränderungen wies schwere, mit Wassersucht einhergehende Kachexie auf. Bei den letztgenannten drei Fällen war der bakteriologische Befund im Darm negativ, was nach dem oben Gesagten zu erwarten war.

Bei den übrigen 10 Fällen konnte man im Milzausstrich Tropicaparasiten feststellen, als Zeichen einer floriden oder vielleicht auch latenten Infektion; hier war es manchmal schwer zu sagen, ob die Malaria als Todesursache oder als Nebenbefund zu betrachten ist. Sämtliche zeigten eine mehr oder weniger schwere Anämie, die natürlich für den letalen Ausgang nicht gleichgültig war; bei einigen waren die Parasiten im Milzausstrich so zahlreich, daß man an einen floriden Prozeß denken mußte (z. B. Fall 43). Den klinischen Aufzeichnungen war nicht viel über den Verlauf der Malaria zu entnehmen; die Krankheitsdauer schwankt zwischen 1 und 5 Monaten, es war aber nicht immer klar, ob die Ruhrinfektion zugleich stattgefunden hatte. Uberhaupt waren die Angaben über Ruhrsymptome äußerst lückenhaft. Der anatomische Darmbefund wich von dem üblichen Bild der Ruhr nur soweit ab, als infolge der schweren Anämie die Hyperämie der Darmschleimhaut manchmal stark zurücktritt.

In den Lohrbüchern wird als eine besondere Form der Malaria der dysenterische Typus derselben beschrieben, wobei die Darmaffektion auf

${ }^{1}$ Anmerkung bei der Korrektur. Die Mitteilung von Raubitachek (Verhandlungen der Deutschen Pathologischen Gesellschaft. Bd. XV), welche das-' selbe Thema behandelt, wurde mir erst nach AbschluB dieser Arbeit zugänglich. 
eine Verstopfung der Darmkapillaren mit Parasiten und Pigment zurückgeführt wird. Bei meinen Fällen handelte es sich bestimmt um nichts derartiges, sondern um Kombination zweier selbständiger Infektionskrankheiten. Das beweist der meist positive Ruhrbazillenbefund; in den wenigen Fällen; wo dieser verrsagte $(33,34,59)$, war nicht der geringste Unterschied in den Darmveränderungen festzustellen. Dor einzige Fall, bei dem die Veränderungen (Hyperämie, Verschorfungen und Blutungen) nicht diffus, sondern herdförmig angeordnet waren, so daB. man an Embolien hätte denken können, ergab positiven Befund von Ruhrbazillen (Fall 58 ).

Wir haben also gesehen, daß man sowohl bei akuter wic anch bei chronischer Ruhr die anatomische Diagnose durch den Nachweis vou Ruhrbazillen erhärten kann, was in vielen zweifelhaften Fällen von Wert ist. Insbesondere möchte ich hervorheben, daB hier oft der Befund positiv ausfallt, wo die Stuhluntersuchung intra vitam wiederholt ergebnislos war $(78,13,79)$.

In einer Anzahl der Fällo lieferte dagegen die bakteriologische Untersuchuing des Präparates ein negatives Ergebnis. Soweit es sich dabei um Prozosse handelt, die offenbar in Abheilung begriffen sind, oder sogar nur um Spuren und Roste einer abgelaufenen Erkrankung, ist der negative Bofund, wie oben näher ausgeführt wurde, ohne weiteres begreiflich. Dies mag etwa für die Fälle 5, 6, 33, 59, 72, 73, 63, 74, 18, 83, 17, 21, 12 und 76 Geltung haben. Was die übrigen negativen Befunde betrifft, so darf nicht außer acht gelassen werden, daß man bei dem Bearbeiten des Loichenmaterials mit derselben Unzulänglichkeit der bakteriologischen Methódik an kämpfen hat, wie bei der Untersuchung von Dejekten. Zwar liegen hier die Verhältnisse insoweit günstiger, daB die Frist zwischen der Entnahme und Bearbeitung des Materials wegfält, doch ist dieser Vorteil nur scheinbar. Es ist nämlich nicht immer möglich, die Obduktion sofort nach dem Tode vorzunehmen; da aber die Leiche, hauptsächlich im Sommer, ihre Wärme ziemlich langsam abgibt, so macht sich hier der ungünstige Einfluß der Uberwucherung durch Saprophyten eher stärker fuihlbar als bei Stuhlproben, die doch viel schneller abkühlen.

Viel größere Bedeutung für das Gelingen des Ruhrbazillennachweises hat die Möglichkeit, direkt von der erkrankten Schleimhaut abimpfen zu können, unter Ausschlub der kotigen Stuhlmassen, die durch Wasserspiilung sorgfältig entfernt wurden. Ich habe schon oben hervorgehoben, daß es diejenigen Stellen der Darminnenwand sind, die die frisehesten Stadien der Erkrankung bzw. des Razidivs aufweisen, von welchen der. Ruhrbazillennachweis am ehesten gelingt. Nur aus dem manehmal mit- 
erkrankten Ileum sind die Ruhrbazillen meist schwer zu züchten, wofür ich keine Erklärung finde.

In einer Anzahl der Fälle (9) wurden die Platten vom B. proteus iiberwuchert. Ohne mich in Betrachtungen iuber Pathogenität dieser Bakterienart im allgemeinen einzulassen, finde ich es für nötig, diesen Befunden einige Bemerkungen zu widmen. Daß der Proteus in pathologischen Stühlen, vor allem bei der Ruhr, oft gefuriden wird, ist bekannt (Köhler,. Pollak und Müller u. a.). Seine ursächliche Beziehung zu diesem Krankheitsprozeß erscheint aber sehr zweifelhaft. Nach Engel soll er bei der Ruhr die Rolle des sogenannten Nosoparasiten (Hansemann) spielen, d. h. er siedelt sich an der durch Ruhrbazillen krankhaft; veränderten Darmschleimhaut an und trägt seinerseits zur Verschlimmerung des Prozesses bei. Er fand bei zwei zur Obduktion gekommenen Ruhrfällen neben den durch die Ruhr bedingten Erseheinungen eine auffallend sulzig-ödematöse Beschaffenheit; er glaubt diese Abweichung der Einwirkung des Proteus zuschreiben. zu müssen, den er neben anderen Bakterien aus der Darmwand züchtete; Ruhrbazillen wurden dabei nicht gefunden. Ich kann diese Beobachtung nicht bestätigen; meine diesbezüglichen Fälle $(14,20,22,25,27,36,86,87,95)$ unterscheiden sich in dieser. Richtung von dem typischen Bild der Ruhr mit Ausnahme einiger mit sekundärer Darmtuberkulose kombinierten Affektionen. Ubrigens waren auch in zahlieichen anderen als den hier angeführten Fällen einige Proteuskolonien auf den Platten zu finden, oft neben Ruhrbazillen; es hängt nämlich einerseits von der rechtzeitigen Besichtigung der Platten, andererseits von der Ten'denz des betreffenden Proteusstammes zur flächenhaften Ausbreitung $\mathbf{a b}$, ob die ganze Platte iuberwuchert wird oder nicht. Ist das einmal der Fall, so wird natürlich dadurch jede Analyse der übrigen Darmflora vereitelt; die möglicherweise auf der Platte vorhandenen Ruhrkolonien lassen sich dann nicht mehr abimplen, da die große Wachstumsenergie des Proteus deren Reinziichtung unmöglich macht. Manchmal ist es reiner Zufall, ob man rechtzeitig dazu kommt, um noch einige Ruhrkolonien ohne die störende Beimischung. abstechen zu können; als Beispiel kann Fall 65 dienen, wo knapp am Rande des die ganze Platte bedeckenden Proteusrasens eine einzige Ruhrkolonie eben noch unberührt geblieben war. Ich sehe also keinen Grund, den Befunden von Proteus eine andere Bedeutung beizumessen als die, daß er das Auffinden der eventuell auf der Platte vorhandenen Ruhrbazillen verhindert.

Wenn ich also sowohl die Fälle mit dem Befund inagglutinabler ruhrähnlicher Bakterien, deren Bedeutung schwer zu beurteilen ist, wie auch 
jene, wo die Platten vom Proteus überwuchert wurden, von der weiteren Betrachtung ausschließe, so bleibt immerhin eine Anzahl Fälle übrig, we der Befund negativ war. Hier gibt es zwei Möglichkeiten: entweder waren Ruhrbazillen da, haben sich aber dem Nachweis entzogen, oder aber es hatten die betreffenden Darmaffektionen mit Ruhrbazillen gar nichts zu tun, sondern es handelte sich um Erkrankungen mit einer anderen Ätiologie.

Diese zweite Möglichkeit möchte ich noch am ehesten für die Fälle 68 und 85 gelten lassen. Bei dem ersten hatte ich im Verlaufe einer tuberkulösen Koxitis subakute Glomerulonephritis entwickelt, und es ist die Möglichkeit nicht von der Hand zu weisen, daß die vorgefundenen Darmveränderungen (Schwellung, Rötung, Blutungen) nichts anderes als Begleiterscheinungen einer Urämie waren. Bei dem zweiten wichen die Erscheinungen im Darm stark vom üblichen Bild der Rubr ab; sie waren eher als chronische Enteritis aufzufassen, und die Untersuchung wurde hier mehr aufs Geratewohl angestellt; am ehesten hätte man noch an Paratyphus denken können. Weiter gehören hierher drei Fälle $(16,52,56)$ mit sekundärer Darmtuberkulose, wo außerdem ähnliche, an akute katarrhalische Ruhr erinnernde Veränderungen vorgefunden wurden, wie jene auf S. 359 besprochenen, mit dem Unterschied aber, da $B$ hier keine Ruhrbazillen gefunden werden kounten; hier ist an die Möglichkeit zu denken, daß die diffuse Entzündung der Schleimhaut durch die von den Geschwüren sezernierten Toxine verursacht war. Schließlich wäre noch Fall 66 zu erwähnen, bei dem katarrhalische Colitis neben einer schweren Peritonealtuberkulose bestand.

Der Rest der Fälle $(1,26,24,31,88)$ aber bot anatomisch das für chronische rezidivierende $\mathrm{Ruhr}$ durchaus typische. Bild; der bakteriologische Befund war jedoch negativ. Es ist die Frage, ob es sich hier überhaupt um Ruhr handelte. Neben der Ruhr gibt es eine Krankheit, die mit ähnlichen geschwürigen Darmveränderungen einhergeht, die Colitis ulcerosa s. gravis; ihre Atiologie ist unbekannt. Schmidt, der dieses Loiden zum erstenmal als selbständige Krankheit beschrieb, definiert es in folgender Weise:

„Unter der Bezeichnung Colitis ulcerosa od. gravis versteht man gewisse Formen schwerer, gewöhnlich langsam sich entwickelnder, manchmal aber akut einsetzender, oft mit Fieber und nicht selten auch mit geschwürigem Zerfall einhergehenden Zustände der Dickdarmschleimbaut, welche bald das ganze Organ, bald nur die tieferen Abschnitte (Flexura, Sigma und Rectum) betreffen und von der Schleimbaut auf die übrigen Wandschichten des Organs überzugreifen pflegen.

Die entzündete Schleimhaut kann in größerer oder geringerer Ausdehnung geschwürig zerfallen und dann besteht kein greifbarer Unterschjed mehr gegenüber der Dysenterie, mit der sie das Vorkommen 
schwerer Allgemeinerscheinungen (hohes Fieber, Anämie, Prostation) und metastatischer Infekte (Gelenksschwellungen, Venenthrombosen) teilt. Sie unterscheidet sich prinzipiell von ihr durch das Fehlen spezifischer Krankheitserreger und durch die oft schleichende Entstehung."

Nun ist aber „das Fehlen spezifischer Krankheitserreger" ein rein negatives Merkmal, das zur Abgrenzung eines 'Krankheitsbegriffes nicht ausreicht; es gilt doch in der Bakteriologie als allgemein anerkannte Regel, daß negative Befunde um so weniger beweisen, je ungenauer die angewandte Methode arbeitet. $\mathrm{DaB}$ aber dies für den Nachweis von Ruhrbazillen in hohem Maße gilt, wurde, oben ausführlich besprochen. Strauss, Dünner, Ehrmann, Gluzinski sowie Dressel und Marchand haben bei Fällen von Colitis ulcerosa im Krankenserum Agglutinine fïr Ruhrbazillen festgestellt; wenn auch die Bedeutung der Agglutination wenigstens für Pseudodysenteriebazillen, fraglich ist; so hat es doch den Ansehein, daß die Colitis ulcerosa in den meisten Fällen, wenn auch vielleicht nicht immer, das Spätstadium eines ursprünglich unerkannten Ruhrinfekts darstellt (Umber). Ich habe bereits oben für diejenigen meiner Fälle, bei welehen abheilende Darmveränderungen gefunden worden sind, diese Auffassung gelten lassen. Aber auch für die zuletzt angeführten Fälle scheint mir die Annahme viel wahrscheinlicher zu sein, daß es sich hier um chronische rezidivierende Ruhr gehandelt habe, der Nachweis der Ruhrbazillen aber wegen der unzulänglichen Methodik mißlang. War doch auch in den Fällen mit positjvem Befund die Zahl der Ruhrkolonien auf den Platten manchmal recht gering. Insbesondere möehte ich darauf hinweisen, daß die Therapie auf die Auffindbarkeit der Ruhrbazillen nicht ohne Einflub bleibt, was auch Schweriner betont.

Das gilt vor allem für den Fall 31, der die schwersten Darmveränderungen aufwies, die ich je gesehen habe: die unteren zwei Drittel der Dickdarmschleimhant waren mit tiefen sinuösen Geschwüren durchlöchert, die im Rectum und hauptsächlich im Sigma zu größeren Flächen konfluierten; die ganze Innenwand des Darmrohres war mit zusammenhängenden, schmutziggrauen, trockenen Pseudomembranen bedeckt, die sich vom Rectum bis ins Ileum erstreckten und auch die Geschwüre auskleideten. Der Fall wurde noch kurz vor dem Tode mit ausgiebigen Darmspülungen mit Tamalbin behandelt; damit ist wahrscheinlich der negative bakteriologische Befund zu erklären.

Die übrigen Fälle wurden mit Bolus oder Tierkohle behandelt, was nach Schweriner und auch nach meiner Erfahrung bei Stuhluntersuchungen ebenfalls nicht gleichgiiltig ist, wenn auch bei direktem Abimpfen von der Darmwand der störende Einfluß in nicht so hohem Maße sich fühlbar macht wie bei Stuhlproben. 
Auf eine Besonderheit meiner Befunde möchte ich noch hinweisen, die vielleicht mit dem eben Besprochenen im Zusammenhang steht: dạs ist die niedrige Zahl der Fälle mit Shiga-Krusebazillen; es waren insgesamt fünf Fälle; einer davon (92) betraf akute, die übrigen $(3,23,61,91)$ chronische Erkrankungen. Für das Uberwiegen der Pseudodysenteriebazillen in den chronischen Formen kann es zweierlei Erklärung geben: entweder zeigen die durch diese Abart verursachten Erkrankungen größere Neigung zum chronischen Verlauf (Gruber und Schädel), es würde sich also um eine besondere Eigenschaft der Pseudoruhrbazillen handeln, oder aber, was viel wahrscheinlicher erscheint, entziehen sich die Shiga-Krusebazillen wegen ihrer größeren Emplindlichkeit leichter dem bakteriologischen Nachweis. Damit wäre vielleicht mancher der negativen Béfunde zu erklären; daß diese Annahme nicht von der Hand zu weisen ist, beweist Fall 84, wo trotz des Vorhandenseins von schweren rezidivierenden Prozessen aus 'dem Darm keine Ruhrbazillen gezüchtet werden konnten; drei Wochen vorher wurden aber im Stuhle Shiga-Krusebazillen nachgewiesen. (Es ist uibrigens der einzige Fall, wo die bakteriologische Untersuchung an der Leiche im Gegensatz zur Stuhluntersuchung versagte.) Irgendwelche Besonderheiten im pathologisch-anatomischen Bilde der durch Shiga-Krusebazillen verursachten Erkrankungen konnte ich nicht feststellen. $\mathrm{Ob}$ die Pseudoruhrbazillen im allgemeinen leichtere Formen der Ruhr zu erzeugen pflegen, wie Kruse behauptet, entzieht sich meinem Urteil; sicher ist aber, daß sie nicht selten die allerschwersten Darmveränderungen veranlassen kồnnen.

Die Ergebnisse meiner Untersuchungen lassen sich etwa in folgendrr Weise zusammenfassen:

1. Aus dè erkrankten Darmschleimhaut von Ruhrleichen lassen sich in den meisten Fällen Ruhrbazillen züchten.

2. Die Ätiologie mancher Darmaffektionen, deren Zugehörigkeit zur Ruhr nach dem anatomischen Befunde fraglich erseheint, kann auf diese Weise aufgeklärt werden. Es zeigt sich dabei, daß die Ruhr in Form einer ganz leichten katarrhalischen Entzindung der Dickdarmschleimhaut auftreten kann.

3. Chronische Ruhr weist in den weitaus meisten Fällen einen rezidivierenden Charakter auf. Diese. Rezidive sind spezifischer Natur; es lasse sich aus den ergriffenen Schleimhautpartien Ruhrbazillen züchten. Deshalb sind chronisch Ruhrkranke stets als ansteckungsgefährlich zII betrachten. 
4. Für die Unterhaltung der: dysenterischen Prozesse bei ehronischer Ruhr scheinen die Schleimzysten and submukösen Abszesse von Bedeutung zu sein, da sich in ihnen während der Latenz Ruhrbazillen erhalten können.

5. Die im Verlaufe der Tuberkulose, Malaria und septischen Erkrankungen auftretenden Durchfälle sind sehr oft durch unerkannt gebliebene Ruhr bedingt.

Beendet im Juni 1919. 
Untersuchungen am path.-Anat. Material von Bazillenruhr. 383

\section{Literaturverzeichnis.}

A be, Arch. f. Hyg. Bd. LXV.

Adelheim, Deutsche med. Wochenschr. 1918. Nr. 42. S. 1176.

Alter, Ebenda. 1015. Nr. 5.

Amako, Diese Zeitschrift. Bd. LX.

Arneth, Berliner klin. Wochenschr. 1916. Nr. 18.

Aronson, Med. Klinik. 1915. Nr. 49.

Aveline, Boycott und Macdonald, zitiert nach Lentz.

Baerthlein, Münchner med. Wochenschr. 1916. Nr. 44; Centralbl. f. Bakt. Bd. LXXVII; Arb. a. d. Kais. Ges.-Amt. Bd. XL.

Bauch, Centralbl. f. Bakt. Bd. LXXXI.

Beinther, Deutsche med. Wochenschr. 1917. Nr. 48.

Beitzke, Berliner klin. Wochenschr. 1917. Nr. 26; Centralbl. f. Bakt. Bd. LXIV.

Beneke, Münchner med. Wochenschr. 1917. Nr. 39.

Bernhardt, zitiert nach Lientz.

Boehnke, zitiert nach Lentz.

Breinl, Wiener klin. Wochenschr. 1917. Nr. 22.

Brückner, zitiert nach Ghon und Roman.

Bruns, Berliner klin. Wochenschr. 1918. Nr. 3 (Vereinsber.).

Busson, Centralbl. t. Bakt. Bd. LVII.

Cahn, Berliner klin. Wochenschr. 1916. Nr. 24.

Cornil et Ranvier, Manuel d'histol. patholog. II. edit. Vol, III. T. I.

Czaplews ki, Deutsche med. Wochenschr. 1917. Nr. 43.

Ditthorn, Centralbl. $f$. Bakt. Bd. LXVII.

Ditthorn und Neumark, Ebenda. Bd. LXVII.

Dopter, Arch. de med. experim. T. XIX.

Dorendorf und Kolle, Deutsche med. Wochenschr. 1916, Nr. 19.

Doerr, zitiert nach Lentz.

Dresel und Marchand, Diese Zeitschrift. Bd. LXXVI.

Dünner, Berliner klin. Wochenschr. 1915. Nr. 46; Ther. d. Gegenw. 1917. Nr. 5.

Dünner und Lauber, Berliner klin. Wochenschr. 1916. Nr. 47.

Duval und Basselt, zitiert nach Ghon und Roman.

Ebeling, Diese Zeitschrift. Bd. LXXIV.

Eckert, zitiert nach Lontz.

Egyedi, Wiener klin. Wochenschr. 1917. Nr. 21. 
Egyedi und Kulka, Ebenda. 1915. Nr. 38.

Ehrmann, Berliner klin. Wochenschr. 1916. Nr. 48.

Eisenberg, Wiener klin. Wochenschr. 1918. Nr. 4.

Engel, Münchner med. Wochenschr. 1917. Nr. 45.

Falta und Kohn, Wiener klin. Wochenschr. 1915. Nr. 22.

Flatzek, Deutsche med. Wochenschr. 1917. Nr. 7.

Flexner, zitiert nach Shiga.

Ford, zitiert nach Kruse.

Fraenke 1, Deutsche med. Wochenschr. 1915. Nr. 40.

Friedmann, Gentralbl. f. Bakt. Bd. LXXVII.

Friedemann und Steinbock, Deutsche med. Wochenschr. 1916. Nr. 8. Friedrich, Ebenda. 1917. Nr. 51.

Galambos, Wiener klin. Wochenschr. 1918. Nr. 14.

Gaethgens, Zeitschr. $f$ : İmmunitätsforschung. Bd. XII.

Gehrmann, Deutsche med. Wochenschr. 1918. Nr. 37.

Ghon und Roman, Ebenda. 1915. Nr. 22.

Gies kiewicz, Centralbl. f. Bakt. Bd. LXXVIII.

Gildemeister, Ebenda. Bd. LXXVIII und LXXIX.

Gildemeister und Baerthlein, Ebenda. Bd. LXVII.

Gluzinski, Wiener. klin. Wochenschr. 1917. Nr. 18.

Gräf, Diese Zeitschrift. Bd. LIV.

Gruber und Schaedel, Minnchner med. Wochenschr. 1918. Nr. 35 ; Centralbl. $t$. Bakt. Bd. LXXXI.

Gruidmann, Berliner klin. Wachenschr. 1915. Nr. 42-44.

Hamburger, Ebenda. 1917. Nr. 23.

Hamburger und Bauch, Ebenda. 1917. Nr. 32.

Handmann, Deutsche med. Wochenschr. 1916. Nr. 30.

Hansemann, Berliner klin. Wochenschr. 1916. Nr. 44.

Hawkins, zitiert nach Lentz.

Helly, zitiert nach Galambos.

Herford, zitiert nach Herxheimer.

Herxhei mer, Berliner klin. Wochenschr. 1916. Nr. 24.

Hetsch, zitiert nach Lentz.

Hesse, Deutsche med. Wochenschr. 1916. Nr. 23.

Heubner, Ziemssens Handb. d. spez. Path. u. Therap. II. Aufl. 1887.

Hirsch, Deutsche med. Wochenschr. 1915. Nr. 40.

Hiss und Russel, zitiert nach Shiga.

Hutt, Diese Zeitschrift. Bd. LXXIV.

Jakobitz, Berliner klin. Wochenschr. 1916, Nr. 26.

$J$ ürgens, Med. Klinik. 1916. Nr. 51.

Kathe, Deutsche med. Wochenschr. 1918. Nr. 18.

Karlinski, zitiert nach Ebeling.

Ke ck, Diese Zeitschrift. Bd. LXXXVI.

Kem p, Ebenda. Bd. LVII.

Ke u per, Mïnchner med. Wochenschr. 1917. Nr. 36

Kindborg, Berliner klin. Wochenschr. 1917. Nr. 18.

Kisskalt, Deutsche med. Wochenschr. 1915. Nr. 20.

Knox und Schorrer, zitiert nach Lentz. 
Untersuchungen am path.-anat. Material von Bazillenruir. 385

Koch, Deutsche med. Wochenschr. 1916. Nr. 17.

Köhler, Münchner med. Wochenschr. 1918. Nr. 27.

Köhlisch, Berliner klin. Wochenschr. 1916. Nr. 14.

Kohnrich, Diese Zeitschr. Bd. LX.

Korczynski, Berliner klin. Wochenschr. 1915. Nr. 46.

Kraege l, Centralbl. f. Bakt. Bd. LVIII.

Kruse, Deutsche med. Wochenschr. 1915. Nr. 36; Münchner med. Wochensch rift. 1917. Nr. 40.

Kruse, Rittershaus, Kemp und Metz, Diese Zeitschrift. Bd. LVII.

Kuenen, Arch. f. Schiffs. u. Tropenhyg. XIII. Beih. Bd. VII.

Kuhn, Med. Klinik. 1916. Nr. 30; Arch.f. Hyg. Bd. LXXXVI; Centralblalt f. Bakt. Bd. LXXX.

Kuhn und Eveling, Zeitschr. f. Immunitätsforschung. Bd. XXV.

Kuhn, Yoithe und Gildemeister, Arbeiten a. d. Kais. Gesundh.-Amt.

Bd. XXXI und XXXVIII.

Kulka, Med. Klinik. 1917. Nr. 48.

$\mathrm{K}$ üster, zitiert nach Lentz.

Kutscher, Münchner med. Wochenschr. 1915. Nr. 36.

Lampl, Wiener klin. Wochenschr. 1918. Nr. 30.

Lingels hei m, Centralbl. f. Bakt. Bd. LXVIII.

Lentz, Handb. d. path. Mikroorg. Kolle und Wassermann. II. Aufl.

Bd. III.

Lentz und Kantorowitz, zitiert nach Lentz.

Loewenthal, Diese Zeitschr. Bd. LXXII.

v. Loghem, zitiert nach Lentz.

Löhlei n, Med. Klinik. 1917. Nr. 6, 17, 18, 20, 21.

Lucksch, Centralbl. f. Bakt. Bd. XXXIV.

Manteufe 1, Diese Zeitschr. Bd. LXXIX.

Markwald, zitiert nach Ghon und Roman.

Marx, Berliner klin. Wochenschr. 1915. Nr. 50.

M a yer, O., Centralbl. f. Bakt. Bd. LXVI.

Me yer, F., Berliner klin. Wachenschr. 1916. Nr. 34-40.

Menzer, Berliner klin. Wochenschr. 1915. Nr, 48.

Menzer, Ebenda. 1915. Nr. 48.

Mühlmann, Arch. f. Hyg. Bd. LXIX.

Müller, Centralbl. f. Bakt. Bd. LVIII.

Negri und Pane, zitiert nach Lentz.

Neukirch, Diese Zeitschrift. Bd. LXXXV.

Nowicki, Berliner klin. Wochenschr. 1917. Nr. 52.

Orth, Lehrbuch. 1887; Berliner klin. Wochenschr. 1918. Nr. 29.

Otto, zitiert nach Ebeling.

Popper, Wiener klin. Wochenschr. 1917. Nr. 45.

Pfuhl, zitiert nach Lentz.

Pollak und Müller, Wiener klin. Wochenschr. 1917. Nr. 49.

Pr ym, Deutsche med. Wochenschr. 1918. Nr. 20.

Rim pau, Arb. a. d. Kais. Ges.-Amt. Nr. 38.

Rokitanski, Lehrbuch.

Rose, Berliner klin, Wochenschr. 1916. Nr. 24.

Zeitschr. t. Hygiene. $\mathrm{XC}$ 
386 Hermann S̈IKL: Untersuchungen am path.-ANat. Material usw.

Rosenthal, zitiert nach Kraegel.

Rum pel, Münchner med. Wochenschr. 1915. Nr. 6.

Rumpel und Knack, Deutsche med. Wochenschr. 1916. Nr. 44-47.

Sachs und Mücke, Med. Klinik. 1917. Nr. 6.

Salus, Wiener klin. Wochenschr. 1915. Nr. 41.

Sangiorgi und Bangio annini, Centralbl. f. Bakt. Bd. LXIX.

Schiemann, Diese Zeitschrift. Bd. LXXXIr.

Schmidt, Mitt. d. Grenzgeb. Bd. XXVII.

Sohmidt und Kaufmann, Münchner med. Wochenschr, 1917. Nr. 23.

Schmitz, Münchner med. Wochenschr. 1917. Nr. 49; Centralbl. f. Bakt. Bd. LXXXI.

Schroeder, Deutsche med. Wochenschr. 1917. Nr. 37.

Schroeder und Umnus, Med. Klinik. 1915. Nr. 23.

Schroeter und Gutjahr, Centralbl. f. Balt. Bd. LVIII.

Schürer und Wolff, Deutsche med. Wochenschr. 1918. Nr. 33.

Schweriner, Berliner klin. Wochenschr. 1918. Nr. 10.

Seligman, Centralbl. f. Bakt. Bd. LXXIX.

Seligmann und Cossmann, Münchner med. Wochenschr. 1915. Nr. 51.

Shiga, Diese Zeitschr. Bd. LX; Deutsche med. Wochenschr. 1901. Nr. 43.

Singer, Münchner med. Wochenschr. 1915. Nr. 16.

Soldin, Berliner klin. Wochenschr. 1915. Nr. 43.

Sonne, Centralbl. f. Bakt. Bd. LXXV und LXXVI.

Starck, Münchner med. Wochenschr. 1917. Nr. 49.

Sternberg, Wiener klin. Wochenschr. 1916. Nr. 40; Zieglers Beiträge.

Bd. LXIV.

Strauss, Deutsche med. Wochenschr. 1915. Nr. 36; Ther. d. Qegenwart. 1917. Nr. 16.

Strong, zitiert nach Shiga.

Umber und Friedemann, Deutsche med. Wochenschr. 1918. Nr. 49.

Ungermann und Jötten, Med. Klinik. 1918. Nr. 15.

Vaillard und Dopter, zitiert nach Kraegel.

Wagner, Münchner med. Wochenschr. 1916. Nr. 18.

Wells und Scott, zitiert nach Herxheimer.

Weltmann, Deutsche med. Wochenschr. 1918. Nr. 1.

Westenhöfer, Berliner klin. Wochenschr. 1918. Nr. 47.

Winter, Diese Zeitschrift. Bd: LXX.

Wolf, zitiert nach Kruse. 